\title{
Quantum chemical studies on tautomerism, isomerism and deprotonation of some 5(6)-substituted benzimidazole-2-thiones
}

\author{
C. Öğretir ${ }^{*}$, I. İ. Öztürk, and N. F. Tay \\ Eskişehir Osmangazi University, Faculty of Arts \& Sciences, Chemistry Department, \\ 26040 Eskişehir, Turkey \\ E-mail: cogretir@ogu.edu.tr
}

\begin{abstract}
Acidity constants, $\mathrm{pK}_{\mathrm{a}}$ values, tautomeric and isomeric equilibrium constants, $\mathrm{K}_{\mathrm{T}}$ and $\mathrm{K}_{\mathrm{eq}}$. values, of some 5(6)-substituted benzimidazole-2-thiones and related fixed models, in which the possibility of proton migration is eliminated by replacing the mobile proton with methyl group, were calculated using semi-empirically computed physical and thermodynamic parameters. Full geometry optimization was carried out using semi-empirical AM1, PM3 and PM5 methods. The theoretically calculated acidity constants were compared with the experimental values and a reasonable correlation was observed.
\end{abstract}

Keywords: Benzimidazole-2-thione; relative stability; acidity constant; proton affinity; tautomerism

\section{Introduction}

Due to the antagonist effect of benzimidazole derivatives towards purine compounds, the investigation of benzimidazole-2-thiones has been a matter of interest for a long time. ${ }^{1,2}$ Wolley had observed that benzimidazole inhibits the growth of several kinds of yeasts and bacteria. He also proved that the inhibition could be completely eliminated by the addition of aminopurines. ${ }^{3}$ Klotz and Mellody demonstrated that yeast nucleic acid reversed the inhibitory effect of benzimidazole on the growth of the bacterium, Eschericha coli. ${ }^{4}$ Some benzimidazole and imidazole derivatives were investigated earlier both by experimental and theoretical method by our research group. ${ }^{5-13}$

An understanding of the tautomeric and isomeric equilibrium of heterocycles, especially thiol-thione, helps in understanding many areas of chemistry and biochemistry, such as rationalization of physical and chemical properties and quantitative reactivity of heterocycles, ${ }^{14-}$ 20 the variation of intrinsic stabilities, solvent effects, ${ }^{21-23}$ as a test of aromaticity, ${ }^{24-26}$ and enzymatic catalysis and receptor interactions. ${ }^{27}$ Therefore, we believe that $5(6)$-substituted 
benzimidazole-2-thione derivatives deserve a detailed theoretical study and four derivatives of 5(6)-substituted 2-mecapto benzimidazole along with their half model molecules, in which the migration of one proton was eliminated by replacing one of the mobile hydrogen atoms with a methyl group, were investigated in the present work. Since the protonation of these four compounds had been studied already, ${ }^{5,6}$ we have concentrated now on deprotonation. The nomenclature and formulation of the compounds studied are depicted in Table 1.

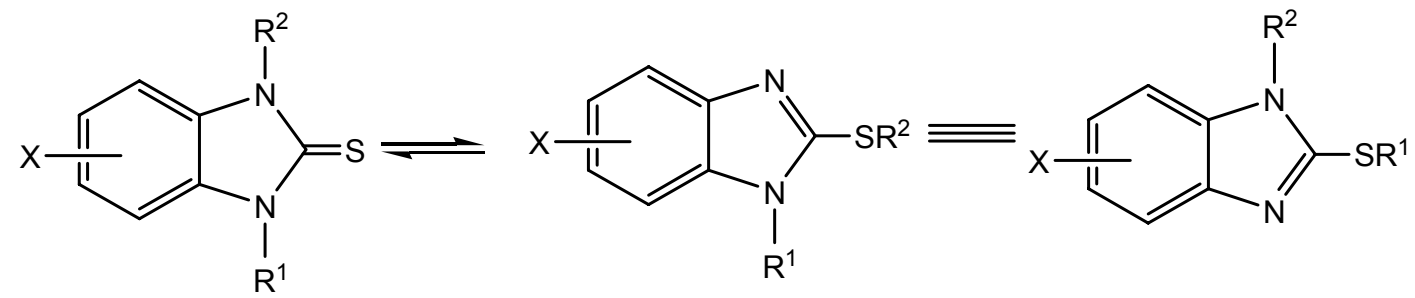

thione form thiol forms

parent molecules : $\mathrm{R}^{1}=\mathrm{R}^{2}=\mathrm{H}$

model molecules: $\mathrm{R}^{1}=\mathrm{CH}_{3} \mathrm{R}^{2}=\mathrm{H}$ or $\mathrm{R}^{1}=\mathrm{H} \mathrm{R}^{2}=\mathrm{CH}_{3}$

\section{Computational methods}

Theoretical calculations were carried out at the restricted Hartree-Fock level (RHF) using AM1, PM3 and PM5 semi-empirical SCF-MO methods in the MOPAC 2002 program, ${ }^{28}$ implemented on an Intel Pentium IV PC computer, using a relative permittivity of 78.4 corresponding to water. The solvent effect was included in the geometry optimizations following the 'COnductorlike Screening Model' $(\mathrm{COSMO})^{20}$ implemented in MOPAC 2002. All the structures were optimized to a gradient norm of $<0.1$ in the gas phase and 0.1-1.0 in the aqueous phase as commonly accepted, using the eigenvector method (EF). The absolute entropies of all structures were calculated from a complete vibration analysis. Enthalpies were corrected to free energies using calculated entropies. Initial estimates of the geometry of all the structures were obtained by a molecular mechanics program (CS ChemOffice Pro for Windows), ${ }^{30}$ followed by full optimization of all geometrical variables (bond lengths, bond angles and dihedral angles), without any symmetry constraint, the semi-empirical AM1, PM3 and PM5 quantum chemical methods in the MOPAC 2002 programs. 
Table 1. Nomenclature of molecules studied

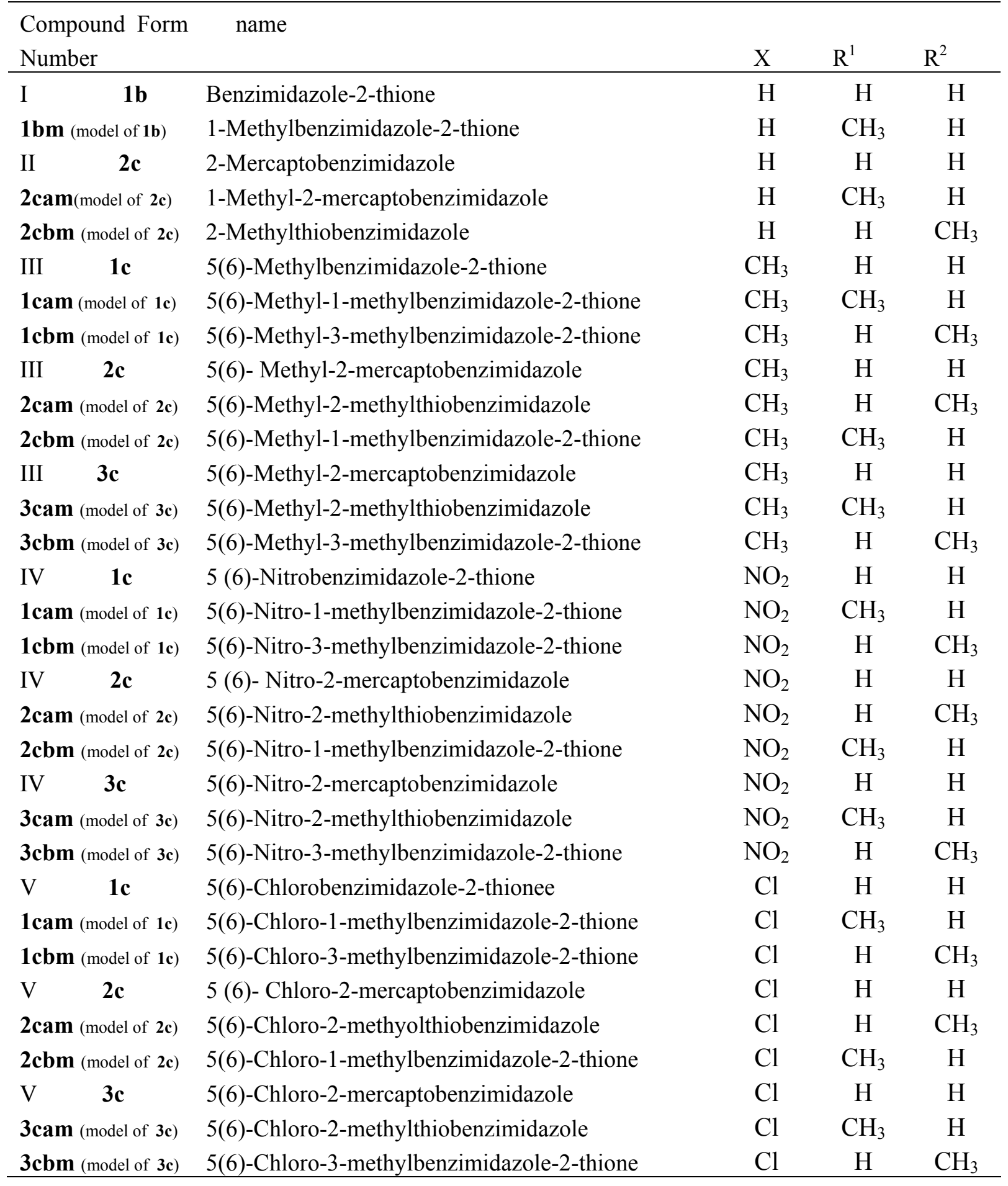




\section{Result and Discussion}

The nomenclature and computed physical and thermodynamic parameters for the studied compounds are depicted in Tables 2-8. We have attempted to evaluate the results obtained as follows.

\section{Relative Stability, Tautomerism and Stereoisomerism}

Among the other possible conformers of mercapto bezimidazoles, with the exception of molecule $\mathbf{I}$ in which $\mathrm{R}^{1}=\mathrm{R}^{2}=\mathrm{H}$ and $\mathrm{X}=\mathrm{H}$, the syn and anti forms are identical, only the anti (i.e. dihedral angle $1234=180^{\circ}$ ) and syn (dihedral angle $1234=0^{\circ}$ ) conformers (Scheme 1) were considered in the present work.

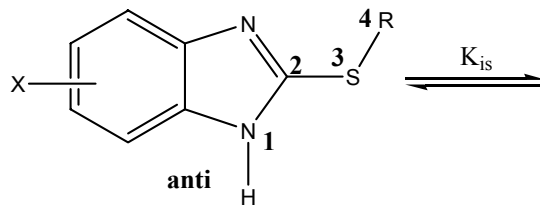

(a)

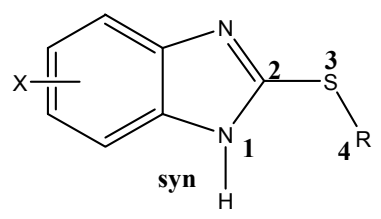

(s)

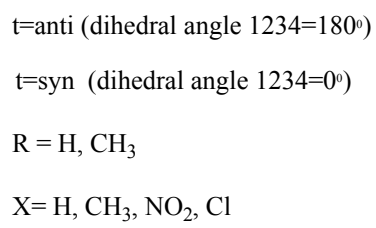

Scheme 1. The anti and syn form for studied molecules.

Using the aqueous phase, AM1, PM3 and PM5 calculated physical and thermodynamic parameters (Table 2) semi-empirical isomerisation and tautomerisation equilibrium constants for 5(6)-substituted benzimidazole-2-thione derivatives along with their fixed models are given Table 3 and Table 4 respectively.

The gas-phase semi-empirical calculated relative stability data for tautomers and isomers along with their fixed models are given Table 5.

As can be seen from Table 3 and Scheme 2, when a potential mercapto group is located at C2 of benzimidazole and its two half models (i.e. one of the mobile hydrogen atoms replaced by a methyl group) the tautomeric equilibrium constants indicate that thione forms I 1c, I 1 cam and I $\mathbf{1 c b m}$ are predominant over thiol forms I 2c, I $2 \mathbf{c a m}$ and I $2 \mathbf{c b m}$ with all methods. 


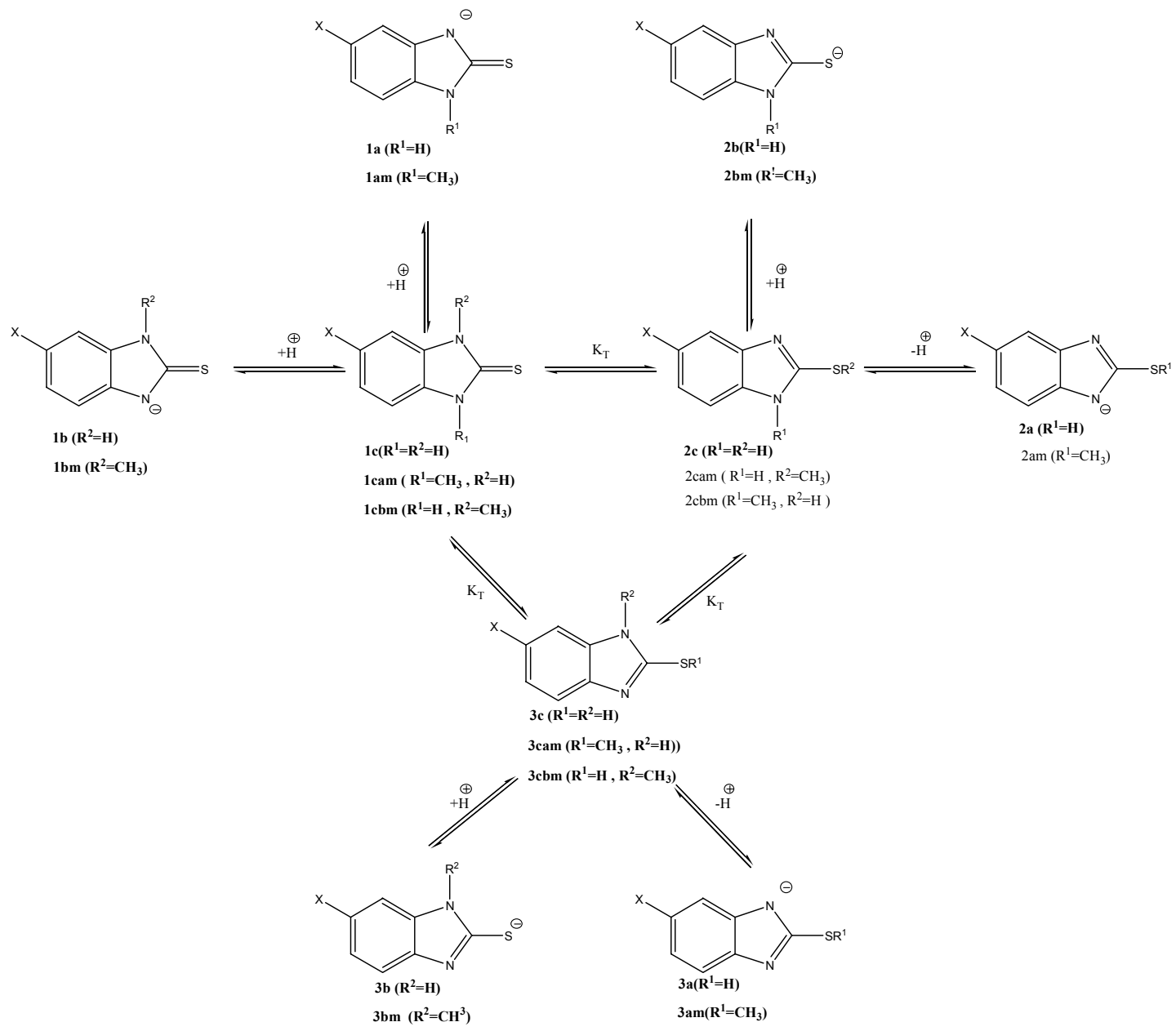

Scheme 2. The deprotonation process and tautomeric forms for 5(6)-substituted benzimidazole2-thione derivatives $\left(\mathrm{X}=\mathrm{H}, \mathrm{CH}_{3}, \mathrm{NO}_{2}, \mathrm{Cl}\right)$.

Table 2. Aqueous phase AM1, PM3 and PM5 calculated thermodynamic data of the studied molecules $(\varepsilon: 78.4)$

\begin{tabular}{|c|c|c|c|c|c|c|c|c|c|c|c|}
\hline Compound & $\begin{array}{c}\Delta \mathbf{H}_{\mathrm{f}} \\
(\mathrm{kcal} / \mathrm{m} \\
\text { ol) } \\
\end{array}$ & $\begin{array}{c}\Delta \mathrm{S} \\
(\mathrm{cal} / \mathrm{mol} \\
\mathrm{K}) \\
\end{array}$ & $\begin{array}{c}\Delta G_{\mathrm{f}} \\
(\mathrm{kcal} / \mathrm{mo} \\
\mathrm{l}^{\mathrm{a}} \\
\end{array}$ & $\begin{array}{c}\text { Compou } \\
\text { nd } \\
\end{array}$ & $\begin{array}{c}\Delta \mathbf{H}_{\mathrm{f}} \\
(\mathrm{kcal} / \mathrm{m} \\
\mathrm{ol}) \\
\end{array}$ & $\begin{array}{c}\Delta \mathrm{S} \\
(\mathrm{cal} / \mathrm{mol} \\
\mathrm{K}) \\
\end{array}$ & $\begin{array}{c}\Delta G_{\mathrm{f}} \\
(\mathrm{kcal} / \mathrm{mo} \\
\mathrm{l})^{\mathrm{a}} \\
\end{array}$ & $\begin{array}{c}\text { Compou } \\
\text { nd } \\
\end{array}$ & $\begin{array}{c}\Delta \mathbf{H}_{\mathrm{f}} \\
(\mathrm{kcal} / \mathrm{m} \\
\mathrm{ol}) \\
\end{array}$ & $\begin{array}{c}\Delta \mathrm{S} \\
(\mathrm{cal} / \mathrm{mol} \\
\mathrm{K}) \\
\end{array}$ & $\begin{array}{c}\Delta G_{\mathrm{f}} \\
(\mathrm{kcal} / \mathrm{mo} \\
\mathrm{l})^{\mathrm{a}} \\
\end{array}$ \\
\hline AM1 & & & & & & & & & & & \\
\hline \multicolumn{12}{|c|}{$\mathbf{X}=\mathbf{H}$} \\
\hline $1 \mathrm{a}$ & -36.26 & 85.85 & -61.84 & $2 c(a)$ & 61.49 & 88.30 & 35.18 & $2 \operatorname{cam}(s)$ & 66.71 & 94.33 & 38.60 \\
\hline $1 \mathrm{c}$ & 53.37 & 85.69 & 27.83 & $1 \mathrm{am}$ & -29.59 & 91.04 & -56.72 & $2 \operatorname{cam}(a)$ & 68.08 & 94.30 & 39.98 \\
\hline $2 a(s)$ & -23.13 & 87.75 & -49.28 & $1 \mathrm{cam}$ & 59.80 & 97.92 & 30.62 & $2 \operatorname{cbm}(s)$ & 55.94 & 97.68 & 26.83 \\
\hline $2 a(a)$ & -23.14 & 87.32 & -49.16 & $2 \operatorname{am}(s)$ & -27.15 & 96.57 & -55.93 & $2 \operatorname{cbm}(a)$ & 56.41 & 98.07 & 27.19 \\
\hline $2 b$ & -36.26 & 85.84 & -61.84 & 2am(a) & -27.15 & 96.35 & -55.86 & & & & \\
\hline
\end{tabular}


$\begin{array}{llllllll}\mathbf{2 c ( s )} & 60.86 & 89.16 & 34.29 & \mathbf{2 b m} & -29.59 & 94.34 & -57.70\end{array}$

\begin{tabular}{|c|c|c|c|c|c|c|c|c|c|c|c|}
\hline \multicolumn{12}{|c|}{$\mathrm{X}=\mathbf{5}(\mathbf{6})-\mathrm{CH}_{3}$} \\
\hline 1a & -43.81 & 90.72 & -70.84 & $3 \mathrm{c}(\mathrm{s})$ & 53.82 & 96.50 & 25.06 & $2 \operatorname{cbm}(s)$ & 59.13 & 104.53 & 27.98 \\
\hline $1 \mathrm{~b}$ & -43.89 & 96.14 & -72.54 & $3 \mathrm{c}(\mathrm{a})$ & 53.16 & 93.98 & 25.15 & $2 \operatorname{cbm}(a)$ & 60.51 & 108.67 & 28.13 \\
\hline $1 \mathrm{c}$ & 45.72 & 91.89 & 18.34 & $1 \mathrm{am}$ & -37.14 & 110.61 & -70.10 & $3 \mathrm{am}(\mathrm{s})$ & -34.69 & 107.03 & -66.58 \\
\hline $2 a(s)$ & -30.71 & 92.65 & -58.32 & $1 \mathrm{bm}$ & -37.20 & 95.89 & -65.78 & 3am(a) & -34.70 & 110.05 & -67.49 \\
\hline 2a(a) & -30.69 & 97.89 & -59.86 & $1 \mathrm{cam}$ & 52.19 & 100.54 & 22.23 & $3 \mathrm{bm}$ & -37.20 & 95.98 & -65.80 \\
\hline $2 b$ & -43.81 & 96.44 & -72.55 & $1 \mathrm{cbm}$ & 52.20 & 96.93 & 23.31 & $3 \operatorname{cam}(s)$ & 48.75 & 107.41 & 16.74 \\
\hline $2 \mathrm{c}(\mathrm{s})$ & 53.24 & 98.69 & 23.83 & $2 \operatorname{am}(s)$ & -34.69 & 106.73 & -66.50 & $3 \operatorname{cam}(a)$ & 48.29 & 109.64 & 15.62 \\
\hline $2 c(a)$ & 53.92 & 93.36 & 26.10 & $2 \operatorname{am}(a)$ & -34.70 & 100.32 & -64.60 & $3 \operatorname{cbm}(s)$ & 60.43 & 104.20 & 29.38 \\
\hline $3 a(s)$ & -30.71 & 94.94 & -59.00 & $2 \mathrm{bm}$ & -37.15 & 98.93 & -66.63 & $3 \operatorname{cbm}(a)$ & 59.17 & 97.78 & 30.03 \\
\hline $3 a(a)$ & -30.69 & 97.89 & -59.86 & $2 \operatorname{cam}(s)$ & 48.37 & 107.05 & 16.47 & & & & \\
\hline $3 \mathbf{b}$ & -43.89 & 94.55 & -72.07 & $2 \operatorname{cam}(\mathbf{a})$ & 48.83 & 107.96 & 16.66 & & & & \\
\hline
\end{tabular}

\begin{tabular}{|c|c|c|c|c|c|c|c|c|c|c|c|}
\hline $1 \mathrm{a}$ & -39.61 & 100.44 & -69.54 & $3 \mathrm{c}(\mathrm{s})$ & 60.01 & 103.78 & 29.08 & $2 \operatorname{cbm}(s)$ & 65.03 & 113.32 & 31.26 \\
\hline $1 b$ & -39.67 & 100.54 & -69.63 & $3 \mathbf{c}(\mathrm{a})$ & 59.32 & 103.52 & 28.47 & $2 \operatorname{cbm}(a)$ & 67.24 & 106.06 & 35.63 \\
\hline $1 \mathrm{c}$ & 53.27 & 102.09 & 22.85 & $1 \mathrm{am}$ & -32.99 & 105.72 & -64.49 & $3 \mathrm{am}(\mathrm{s})$ & -34.01 & 106.87 & -65.86 \\
\hline $2 a(s)$ & -29.77 & 101.06 & -59.89 & $1 \mathrm{bm}$ & -32.97 & 105.81 & -64.50 & 3am(a) & -33.94 & 110.97 & -67.01 \\
\hline $2 a(a)$ & -29.70 & 101.61 & -59.98 & $1 \mathrm{cam}$ & 59.90 & 107.31 & 27.92 & $3 \mathrm{bm}$ & -32.99 & 106.09 & -64.60 \\
\hline $2 b$ & -39.61 & 100.44 & -69.54 & $1 \mathrm{cbm}$ & 59.89 & 107.14 & 27.96 & $3 \operatorname{cam}(s)$ & 54.79 & 111.57 & 21.54 \\
\hline $2 c(s)$ & 58.83 & 103.45 & 28.00 & 2am(s) & -34.01 & 106.47 & -65.74 & $3 \operatorname{cam}(a)$ & 54.22 & 112.50 & 20.70 \\
\hline $2 c(a)$ & 59.52 & 103.94 & 28.55 & 2am(a) & -33.94 & 110.28 & -66.80 & $3 \operatorname{cbm}(s)$ & 67.68 & 105.65 & 36.20 \\
\hline $3 a(s)$ & -29.77 & 101.06 & -59.89 & $2 \mathrm{bm}$ & -32.99 & 108.29 & -65.26 & $3 \operatorname{cbm}(a)$ & 65.56 & 108.58 & 33.20 \\
\hline $3 a(a)$ & -29.70 & 101.61 & -59.98 & $2 \operatorname{cam}(s)$ & 53.75 & 111.76 & 20.45 & & & & \\
\hline $3 b$ & -39.68 & 100.43 & -69.61 & 2cam(a) & 54.41 & 112.26 & 20.96 & & & & \\
\hline \multicolumn{12}{|c|}{$X=5(6)-C l$} \\
\hline $1 \mathrm{a}$ & -43.73 & 92.62 & -71.33 & $3 c(s)$ & 54.68 & 96.52 & 25.92 & $2 \operatorname{cbm}(s)$ & 59.84 & 105.62 & 28.37 \\
\hline $1 b$ & -43.66 & 92.67 & -71.28 & $3 \mathbf{c}(\mathbf{a})$ & 54.00 & 95.89 & 25.42 & $2 \operatorname{cbm}(a)$ & 61.34 & 102.96 & 30.66 \\
\hline $1 \mathrm{c}$ & 47.13 & 93.95 & 19.13 & $1 \mathrm{am}$ & -37.07 & 97.99 & -66.27 & 3am(s) & -35.77 & 103.36 & -66.57 \\
\hline $2 a(s)$ & -31.65 & 94.37 & -59.77 & $1 \mathrm{bm}$ & -37.02 & 97.86 & -66.18 & 3am(a) & -35.76 & 103.61 & -66.64 \\
\hline $2 a(a)$ & -31.65 & 94.42 & -59.79 & $1 \mathrm{cam}$ & 53.64 & 99.11 & 24.11 & $3 \mathrm{bm}$ & -37.02 & 97.85 & -66.18 \\
\hline $2 \mathbf{b}$ & -43.73 & 92.62 & -71.33 & $1 \mathrm{cbm}$ & 53.62 & 99.14 & 24.08 & $3 \operatorname{cam}(s)$ & 49.55 & 104.44 & 18.43 \\
\hline $2 c(s)$ & 53.89 & 95.60 & 25.40 & 2am(s) & -35.77 & 103.25 & -66.54 & $3 \operatorname{cam}(a)$ & 49.04 & 104.82 & 17.80 \\
\hline $2 c(a)$ & 54.59 & 95.04 & 26.27 & 2am(a) & -35.76 & 103.64 & -66.64 & $3 \operatorname{cbm}(s)$ & 61.36 & 105.98 & 29.78 \\
\hline $3 a(s)$ & -31.65 & 94.37 & -59.77 & $2 \mathrm{bm}$ & -37.07 & 100.06 & -66.89 & $3 \operatorname{cbm}(a)$ & 59.93 & 106.42 & 28.22 \\
\hline $3 a(a)$ & -31.65 & 94.50 & -59.81 & $2 \operatorname{cam}(s)$ & 48.93 & 104.14 & 17.90 & & & & \\
\hline $\mathbf{3 b}$ & -43.67 & 92.66 & -71.28 & $2 \operatorname{cam}(a)$ & 49.46 & 104.39 & 18.35 & & & & \\
\hline
\end{tabular}


PM3

\begin{tabular}{crrrrrrrrrrr}
\multicolumn{8}{c}{$\mathbf{X}=\mathbf{H}$} \\
$\mathbf{1 a}$ & -57.72 & 86.75 & -83.57 & $\mathbf{2 c ( a )}$ & 43.35 & 86.91 & 17.45 & $\mathbf{2 c a m ( s )}$ & 42.91 & 95.12 & 14.56 \\
$\mathbf{1 c}$ & 37.04 & 85.99 & 11.41 & $\mathbf{1 a m}$ & -57.09 & 92.33 & -84.60 & $\mathbf{2 c a m ( a )}$ & 44.32 & 93.81 & 16.36 \\
$\mathbf{2 a ( s )}$ & -47.12 & 88.27 & -73.42 & $\mathbf{1 c a m}$ & 37.99 & 92.81 & 10.33 & $\mathbf{2 c b m ( s )}$ & 38.01 & 100.21 & 8.15 \\
$\mathbf{2 a ( a )}$ & -47.10 & 88.34 & -73.43 & $\mathbf{2 a m ( s )}$ & -50.64 & 92.84 & -78.31 & $\mathbf{2 c b m ( a )}$ & 38.92 & 100.02 & 9.11 \\
$\mathbf{2 b}$ & -57.72 & 86.75 & -83.57 & $\mathbf{2 a m ( a )}$ & -50.62 & 100.09 & -80.45 & & & & \\
$\mathbf{2 c ( s )}$ & 42.00 & 90.03 & 15.17 & $\mathbf{2 b m}$ & -57.09 & 96.35 & -85.80 & & &
\end{tabular}

\begin{tabular}{|c|c|c|c|c|c|c|c|c|c|c|c|}
\hline \multicolumn{12}{|c|}{$\mathrm{X}=5(6)-\mathrm{CH}_{3}$} \\
\hline $1 \mathbf{a}$ & -67.11 & 91.44 & -94.36 & $3 c(s)$ & 33.72 & 99.23 & 4.15 & $2 \operatorname{cbm}(s)$ & 33.48 & 104.25 & 2.41 \\
\hline $1 b$ & -67.14 & 95.81 & -95.69 & $3 c(a)$ & 32.56 & 99.90 & 2.79 & $2 \operatorname{cbm}(a)$ & 34.88 & 108.66 & 2.50 \\
\hline $1 \mathrm{c}$ & 27.52 & 97.01 & -1.39 & $1 \mathrm{am}$ & -66.48 & 101.78 & -96.81 & $3 a m(s)$ & -59.94 & 102.58 & -90.51 \\
\hline $2 a(s)$ & -56.42 & 98.60 & -85.80 & $1 \mathrm{bm}$ & -66.49 & 106.83 & -98.33 & $3 a m(a)$ & -59.92 & 101.82 & -90.26 \\
\hline $2 a(a)$ & -56.41 & 97.90 & -85.58 & $1 \mathrm{cam}$ & 28.49 & 103.96 & -2.49 & $3 b m$ & -66.49 & 107.62 & -98.56 \\
\hline $2 \mathbf{b}$ & -67.11 & 95.80 & -95.66 & $1 \mathrm{cbm}$ & 28.48 & 106.48 & -3.25 & $3 \operatorname{cam}(s)$ & 29.47 & 108.68 & -2.92 \\
\hline $2 c(s)$ & 32.57 & 99.40 & 2.95 & $2 \operatorname{am}(s)$ & -59.94 & 102.39 & -90.45 & $3 \operatorname{cam}(a)$ & 28.59 & 103.34 & -2.21 \\
\hline $2 c(a)$ & 33.94 & 97.95 & 4.75 & $2 \operatorname{am}(a)$ & -59.92 & 99.83 & -89.67 & $3 \operatorname{cbm}(s)$ & 34.88 & 106.88 & 3.03 \\
\hline $3 a(s)$ & -56.42 & 99.00 & -85.92 & $2 \mathrm{bm}$ & -66.48 & 99.78 & -96.21 & $3 \operatorname{cbm}(a)$ & 33.47 & 112.03 & 0.09 \\
\hline $3 a(a)$ & -56.41 & 97.90 & -85.58 & $2 \operatorname{cam}(s)$ & 28.57 & 109.61 & -4.09 & & & & \\
\hline $\mathbf{3 b}$ & -67.14 & 94.95 & -95.44 & $2 \operatorname{cam}(a)$ & 29.50 & 103.30 & -1.28 & & & & \\
\hline \multicolumn{12}{|c|}{$\mathrm{X}=5(6)-\mathrm{NO}_{2}$} \\
\hline $1 \mathrm{a}$ & -76.99 & 102.38 & -107.50 & $3 c(s)$ & 24.61 & 100.22 & -5.26 & $2 \operatorname{cbm}(s)$ & 24.52 & 114.62 & -9.64 \\
\hline $1 b$ & -77.19 & 105.13 & -108.52 & $3 c(a)$ & 23.41 & 99.37 & -6.20 & $2 \operatorname{cbm}(a)$ & 25.90 & 105.70 & -5.60 \\
\hline 1c & 20.27 & 96.89 & -8.60 & $1 \mathrm{am}$ & -76.26 & 108.77 & -108.67 & $3 \operatorname{am}(s)$ & -72.53 & 105.97 & -104.11 \\
\hline $2 a(s)$ & -68.80 & 102.56 & -99.36 & $1 \mathrm{bm}$ & -76.38 & 112.66 & -109.95 & $3 \operatorname{am}(a)$ & -72.58 & 106.42 & -104.29 \\
\hline $2 a(a)$ & -68.79 & 102.00 & -99.19 & $1 \mathrm{cam}$ & 21.44 & 102.55 & -9.12 & $3 b m$ & -76.38 & 111.66 & -109.65 \\
\hline $2 b$ & -76.99 & 102.35 & -107.49 & $1 \mathrm{cbm}$ & 21.47 & 106.08 & -10.14 & $3 \operatorname{cam}(s)$ & 20.52 & 108.52 & -11.82 \\
\hline $2 c(s)$ & 23.41 & 99.59 & -6.27 & $2 \operatorname{am}(s)$ & -72.52 & 106.43 & -104.24 & $3 \operatorname{cam}(a)$ & 19.37 & 102.93 & -11.30 \\
\hline $2 c(a)$ & 24.54 & 100.56 & -5.43 & $2 \operatorname{am}(a)$ & -72.61 & 106.53 & -104.36 & $3 \operatorname{cbm}(s)$ & 26.26 & 107.17 & -5.68 \\
\hline $3 a(s)$ & -68.80 & 102.55 & -99.36 & $2 \mathrm{bm}$ & -76.26 & 114.14 & -110.27 & $3 \operatorname{cbm}(a)$ & 24.68 & 108.90 & -7.77 \\
\hline $3 a(a)$ & -68.79 & 102.00 & -99.19 & $2 \operatorname{cam}(s)$ & 19.29 & 102.57 & -11.28 & & & & \\
\hline $\mathbf{3 b}$ & -77.16 & 96.49 & -105.91 & $2 \operatorname{cam}(\mathbf{a})$ & 20.38 & 109.45 & -12.24 & & & & \\
\hline \multicolumn{12}{|c|}{$X=5(6)-C l$} \\
\hline $1 \mathbf{a}$ & -64.85 & 93.63 & -92.75 & $3 c(s)$ & 36.41 & 97.80 & 7.27 & $2 \operatorname{cbm}(s)$ & 36.14 & 102.86 & 5.49 \\
\hline $1 b$ & -64.78 & 94.00 & -92.79 & $3 c(a)$ & 35.24 & 97.08 & 6.31 & $2 \operatorname{cbm}(a)$ & 37.55 & 100.90 & 7.48 \\
\hline 1c & 30.85 & 94.73 & 2.62 & $1 \mathrm{am}$ & -64.20 & 99.49 & -93.85 & $3 \operatorname{am}(s)$ & -58.58 & 100.3 & -88.47 \\
\hline $2 a(s)$ & -55.01 & 95.75 & -83.54 & $1 \mathrm{bm}$ & -64.13 & 105.82 & -95.66 & $3 a m(a)$ & -58.52 & 99.49 & -88.17 \\
\hline
\end{tabular}




\begin{tabular}{|c|c|c|c|c|c|c|c|c|c|c|c|}
\hline $2 a(a)$ & -54.98 & 95.58 & -83.46 & $1 \mathrm{cam}$ & 31.88 & 107.53 & -0.16 & $3 \mathrm{bm}$ & -64.13 & 105.66 & -95.62 \\
\hline $2 b$ & -64.85 & 93.63 & -92.75 & $1 \mathrm{cbm}$ & 31.86 & 104.06 & 0.85 & $3 \operatorname{cam}(s)$ & 32.16 & 106.01 & 0.57 \\
\hline $2 c(s)$ & 35.14 & 97.03 & 6.23 & $2 \operatorname{am}(s)$ & -58.58 & 100.35 & -88.48 & $3 \operatorname{cam}(a)$ & 31.21 & 100.78 & 1.18 \\
\hline $2 c(a)$ & 36.32 & 97.21 & 7.35 & $2 \operatorname{am}(a)$ & -58.52 & 105.58 & -89.98 & $3 \operatorname{cbm}(s)$ & 37.65 & 104.41 & 6.54 \\
\hline $3 a(s)$ & -55.01 & 95.75 & -83.54 & $2 \mathrm{bm}$ & -64.20 & 99.47 & -93.84 & $3 \operatorname{cbm}(\mathbf{a})$ & 36.2 & 102.69 & 5.60 \\
\hline $3 a(a)$ & -54.98 & 95.59 & -83.47 & $2 \operatorname{cam}(s)$ & 31.13 & 100.93 & 1.05 & & & & \\
\hline $3 b$ & -64.78 & 94.00 & -92.79 & $2 \operatorname{cam}(\mathbf{a})$ & 32.09 & 107.33 & 0.11 & & & & \\
\hline
\end{tabular}

PM5

\begin{tabular}{|c|c|c|c|c|c|c|c|c|c|c|c|}
\hline \multicolumn{12}{|c|}{$\mathbf{X}=\mathbf{H}$} \\
\hline $1 \mathbf{a}$ & -72.75 & 87.62 & -98.86 & $2 c(a)$ & 43.54 & 87.63 & 17.43 & $2 \operatorname{cam}(s)$ & 48.48 & 93.39 & 20.65 \\
\hline $1 c$ & 26.26 & 87.59 & 0.16 & $1 \mathrm{am}$ & -66.64 & 99.56 & -96.31 & $2 \operatorname{cam}(a)$ & 50.20 & 93.05 & 22.47 \\
\hline $2 a(s)$ & -50.05 & 88.44 & -76.41 & $1 \mathrm{cam}$ & 32.22 & 98.01 & 3.01 & $2 \operatorname{cbm}(s)$ & 40.95 & 94.58 & 12.77 \\
\hline $2 a(a)$ & -50.06 & 88.45 & -76.42 & $2 \operatorname{am}(s)$ & -50.70 & 92.77 & -78.35 & $2 \operatorname{cbm}(a)$ & 41.13 & 94.30 & 13.03 \\
\hline $2 \mathbf{b}$ & -72.75 & 87.62 & -98.86 & 2am(a) & -50.70 & 98.05 & -79.92 & & & & \\
\hline $2 c(s)$ & 42.77 & 92.14 & 15.31 & $2 \mathrm{bm}$ & -66.64 & 97.96 & -95.83 & & & & \\
\hline \multicolumn{12}{|c|}{$\mathrm{X}=5(6)-\mathrm{CH}_{3}$} \\
\hline $1 \mathrm{a}$ & -80.79 & 92.62 & -108.39 & $3 c(s)$ & 35.37 & 100.18 & 5.52 & $2 \operatorname{cbm}(s)$ & 40.40 & 106.57 & 8.64 \\
\hline $1 b$ & -80.83 & 97.23 & -109.80 & $3 c(a)$ & 34.63 & 97.43 & 5.60 & $2 \operatorname{cbm}(a)$ & 41.50 & 108.37 & 9.21 \\
\hline $1 \mathrm{c}$ & 18.06 & 98.28 & -11.23 & $1 \mathrm{am}$ & -74.69 & 107.22 & -106.64 & $3 a m(s)$ & -58.68 & 104.96 & -89.96 \\
\hline $2 a(s)$ & -58.05 & 99.32 & -87.65 & $1 \mathrm{bm}$ & -74.73 & 101.05 & -104.84 & 3am(a) & -58.70 & 108.20 & -90.94 \\
\hline $2 a(a)$ & -58.04 & 99.35 & -87.65 & $1 \mathrm{cam}$ & 24.09 & 108.88 & -8.36 & $3 b m$ & -74.72 & 100.35 & -104.62 \\
\hline $2 \mathbf{b}$ & -80.79 & 97.33 & -109.79 & $1 \mathrm{cbm}$ & 24.10 & 107.69 & -7.99 & $3 \operatorname{cam}(s)$ & 33.04 & 103.16 & 2.30 \\
\hline $2 c(s)$ & 34.65 & 101.53 & 4.39 & $2 \operatorname{am}(s)$ & -58.64 & 110.60 & -91.60 & $3 \operatorname{cam}(a)$ & 32.87 & 105.12 & 1.54 \\
\hline $2 c(a)$ & 35.46 & 99.79 & 5.72 & 2am(a) & -58.70 & 109.21 & -91.24 & $3 \operatorname{cbm}(s)$ & 41.30 & 102.50 & 10.76 \\
\hline $3 a(s)$ & -58.04 & 99.24 & -87.61 & $2 \mathrm{bm}$ & -74.69 & 107.29 & -106.66 & $3 \operatorname{cbm}(a)$ & 40.38 & 105.76 & 8.86 \\
\hline $3 a(a)$ & -58.04 & 99.35 & -87.65 & $2 \operatorname{cam}(s)$ & 32.89 & 103.50 & 2.05 & & & & \\
\hline $\mathbf{3 b}$ & -80.83 & 96.11 & -109.47 & $2 \operatorname{cam}(a)$ & 33.06 & 102.52 & 2.51 & & & & \\
\hline \multicolumn{12}{|c|}{$\mathrm{X}=5(6)-\mathrm{NO}_{2}$} \\
\hline $1 \mathbf{a}$ & -83.55 & 96.95 & -112.44 & $3 c(s)$ & 34.08 & 100.36 & 4.17 & $2 \operatorname{cbm}(s)$ & 38.97 & 105.69 & 7.47 \\
\hline $1 b$ & -83.49 & 97.06 & -112.41 & $3 c(a)$ & 33.45 & 100.58 & 3.48 & $2 \operatorname{cbm}(a)$ & 40.81 & 106.55 & 9.06 \\
\hline $1 \mathrm{c}$ & 19.57 & 98.29 & -9.72 & $1 \mathrm{am}$ & -77.50 & 106.07 & -109.11 & $3 a m(s)$ & -65.72 & 111.66 & -98.99 \\
\hline $2 a(s)$ & -64.44 & 105.20 & -95.79 & $1 \mathrm{bm}$ & -77.48 & 106.43 & -109.20 & 3am(a) & -65.79 & 110.59 & -98.75 \\
\hline $2 a(a)$ & -64.47 & 105.10 & -95.79 & $1 \mathrm{cam}$ & 25.60 & 108.19 & -6.64 & $3 b m$ & -77.48 & 106.05 & -109.08 \\
\hline $2 b$ & -83.56 & 96.95 & -112.45 & $1 \mathrm{cbm}$ & 25.38 & 117.52 & -9.64 & $3 \operatorname{cam}(s)$ & 31.61 & 110.88 & -1.43 \\
\hline $2 c(s)$ & 33.15 & 100.87 & 3.09 & $2 \operatorname{am}(s)$ & -65.72 & 111.21 & -98.86 & $3 \operatorname{cam}(a)$ & 31.31 & 101.48 & 1.07 \\
\hline $2 c(a)$ & 33.84 & 103.14 & 3.10 & 2am(a) & -65.78 & 108.40 & -98.08 & $3 \operatorname{cbm}(s)$ & 40.73 & 110.87 & 7.69 \\
\hline $3 a(s)$ & -64.44 & 105.23 & -95.80 & $2 \mathrm{bm}$ & -77.50 & 105.81 & -109.03 & $3 \operatorname{cbm}(a)$ & 39.25 & 108.87 & 6.81 \\
\hline
\end{tabular}




$\begin{array}{crrrrrrr}\text { 3a(a) } & -64.47 & 105.10 & -95.79 & \mathbf{2 c a m ( s )} & 30.97 & 103.36 & 0.17 \\ \text { 3b } & -83.52 & 103.07 & -114.23 & \mathbf{2 c a m ( a )} & 31.51 & 103.42 & 0.69\end{array}$

\begin{tabular}{|c|c|c|c|c|c|c|c|c|c|c|c|}
\hline \multicolumn{12}{|c|}{$\mathrm{X}=5(6)-\mathrm{Cl}$} \\
\hline $1 \mathrm{a}$ & -80.58 & 94.76 & -108.82 & $3 \mathrm{c}(\mathrm{s})$ & 36.11 & 100.63 & 6.12 & $2 \operatorname{cbm}(s)$ & 40.98 & 103.64 & 10.10 \\
\hline $1 b$ & -80.45 & 94.78 & -108.69 & $3 \mathbf{c}(\mathbf{a})$ & 35.36 & 99.76 & 5.63 & $2 \operatorname{cbm}(a)$ & 42.10 & 103.82 & 11.16 \\
\hline $1 \mathrm{c}$ & 19.70 & 95.97 & -8.90 & $1 \mathrm{am}$ & -74.51 & 105.51 & -105.95 & $3 \operatorname{am}(s)$ & -59.75 & 107.29 & -91.72 \\
\hline $2 a(s)$ & -58.95 & 95.77 & -87.49 & $1 \mathrm{bm}$ & -74.42 & 99.92 & -104.20 & $\operatorname{3am}(\mathbf{a})$ & -59.74 & 103.59 & -90.61 \\
\hline $2 a(a)$ & -58.95 & 95.78 & -87.49 & $1 \mathrm{cam}$ & 25.72 & 104.84 & -5.52 & $3 \mathrm{bm}$ & -74.42 & 99.91 & -104.19 \\
\hline $2 b$ & -80.58 & 94.76 & -108.82 & $1 \mathrm{cbm}$ & 25.71 & 105.29 & -5.67 & $3 \operatorname{cam}(s)$ & 33.73 & 101.18 & 3.58 \\
\hline $2 c(s)$ & 35.25 & 97.94 & 6.06 & $2 \operatorname{am}(s)$ & -59.75 & 99.01 & -89.25 & $3 \operatorname{cam}(a)$ & 33.48 & 103.35 & 2.68 \\
\hline $2 c(a)$ & 36.04 & 96.75 & 7.21 & $2 \operatorname{am}(a)$ & -59.74 & 103.60 & -90.61 & $3 \operatorname{cbm}(s)$ & 42.23 & 103.88 & 11.27 \\
\hline $3 a(s)$ & -58.95 & 95.77 & -87.49 & $2 \mathrm{bm}$ & -74.51 & 104.80 & -105.74 & $3 \operatorname{cbm}(a)$ & 41.11 & 103.37 & 10.31 \\
\hline $3 \mathbf{a}(\mathbf{a})$ & -58.95 & 95.75 & -87.48 & $2 \operatorname{cam}(s)$ & 33.33 & 101.42 & 3.11 & & & & \\
\hline $\mathbf{3 b}$ & -80.45 & 94.78 & -108.69 & $2 \operatorname{cam}(a)$ & 33.59 & 101.00 & 3.49 & & & & \\
\hline
\end{tabular}

${ }^{\text {a }}$ from $\Delta \mathrm{G}_{\mathrm{f}}=\Delta \mathrm{H}_{\mathrm{f}}-\mathrm{T} . \Delta \mathrm{S}$

H2O - AM1: $\Delta \mathrm{H}_{\mathrm{f}}(\mathrm{kcal} / \mathrm{mol})=-64.39, \Delta \mathrm{S}(\mathrm{cal} / \mathrm{molK})=45.10, \Delta \mathrm{G}_{\mathrm{f}}(\mathrm{kcal} / \mathrm{mol})=-77.83$

PM3: $\Delta \mathrm{H}_{\mathrm{f}}(\mathrm{kcal} / \mathrm{mol})=-58.02, \Delta \mathrm{S}(\mathrm{cal} / \mathrm{molK})=45.00, \Delta \mathrm{G}_{\mathrm{f}}(\mathrm{kcal} / \mathrm{mol})=-71.43$

PM5: $\Delta \mathrm{H}_{\mathrm{f}}(\mathrm{kcal} / \mathrm{mol})=-59.47, \Delta \mathrm{S}(\mathrm{cal} / \mathrm{molK})=44.99, \Delta \mathrm{G}_{\mathrm{f}}(\mathrm{kcal} / \mathrm{mol})=-72.88$

$\mathbf{H}_{3} \mathbf{O}^{+}-\quad$ AM1: $\Delta \mathrm{H}_{\mathrm{f}}(\mathrm{kcal} / \mathrm{mol})=50.61, \Delta \mathrm{S}(\mathrm{cal} / \mathrm{molK})=46.15, \Delta \mathrm{G}_{\mathrm{f}}(\mathrm{kcal} / \mathrm{mol})=36.83$

PM3: $\Delta \mathrm{H}_{\mathrm{f}}(\mathrm{kcal} / \mathrm{mol})=67.33, \Delta \mathrm{S}(\mathrm{cal} / \mathrm{molK})=46.01, \Delta \mathrm{G}_{\mathrm{f}}(\mathrm{kcal} / \mathrm{mol})=53.62$

PM5: $\Delta \mathrm{H}_{\mathrm{f}}(\mathrm{kcal} / \mathrm{mol})=46.51, \Delta \mathrm{S}(\mathrm{cal} / \mathrm{molK})=45.88, \Delta \mathrm{G}_{\mathrm{f}}(\mathrm{kcal} / \mathrm{mol})=32.84$

Table 3. Aqueous phase PM3, PM5 and AM1 calculated tautomeric equilibrium constants $\mathrm{K}_{\mathrm{T}}$ of studied molecules

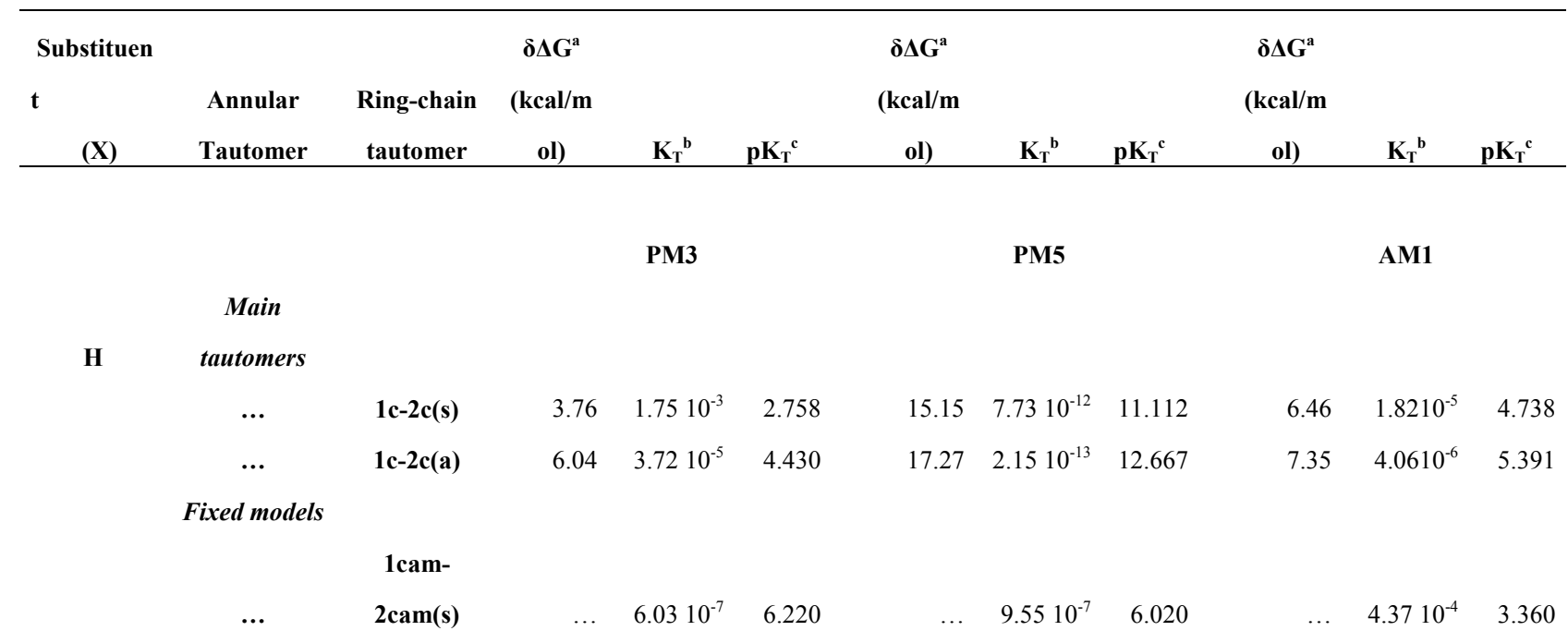




\begin{tabular}{|c|c|c|c|c|c|c|c|c|c|c|}
\hline \multirow[t]{2}{*}{$\cdots$} & 2cam(a) & $\ldots$ & $1.1410^{-4}$ & 3.942 & $\cdots$ & $2.1210^{-5}$ & 4.674 & $\ldots$ & $7.1110^{-4}$ & 3.148 \\
\hline & 1cam- & & & & & & - & & & \\
\hline \multirow[t]{2}{*}{$\cdots$} & $2 \operatorname{cbm}(s)$ & $\ldots$ & $9.5910^{3}$ & -3.982 & $\ldots$ & $3.8410^{12}$ & 12.584 & $\ldots$ & $3.7410^{6}$ & -6.573 \\
\hline & 1cam- & & & & & & - & & & \\
\hline . & $2 \operatorname{cbm}(a)$ & $\ldots$ & $2.0010^{5}$ & -5.302 & $\ldots$ & $8.3010^{13}$ & 13.919 & $\ldots$ & $3.8410^{7}$ & -7.584 \\
\hline
\end{tabular}

Main

5(6)- $\mathrm{CH}_{3}$ tautomers

$\begin{array}{ccccccccccc}\ldots & \mathbf{1 c - 2 c ( s )} & 4.34 & 6.5610^{4} & 3.183 & 15.62 & 3.5010^{-12} & 11.456 & 5.49 & 9.4110^{-5} & 4.027 \\ \ldots & \mathbf{1 c - 2 c ( a )} & 6.14 & 3.1310^{5} & 4.504 & 16.95 & 3.6810^{-13} & 12.434 & 7.76 & 2.0410^{-6} & 5.691 \\ \ldots & \mathbf{1 c - 3 c ( s )} & 5.54 & 8.6410^{5} & 4.063 & 16.75 & 5.1810^{-13} & 12.285 & 6.72 & 1.1810^{-5} & 4.929 \\ \ldots & \mathbf{1 c - 3 c ( a )} & 4.18 & 8.6010^{-4} & 3.066 & 16.83 & 4.5610^{-13} & 12.341 & 6.81 & 1.0110^{-5} & 4.998 \\ \mathbf{2 c ( s ) - 3 c ( s )} & \ldots & 1.20 & 1.3210^{-1} & 0.880 & 1.13 & 1.4810^{-1} & 0.829 & 1.23 & 1.2510^{-1} & 0.902 \\ \mathbf{2 c ( a ) - 3 c ( a )} & \ldots & -1.96 & 2.7410^{1} & -1.438 & -0.13 & 1.24 & -0.093 & -0.94 & 4.93 & -0.693\end{array}$

Fixed models

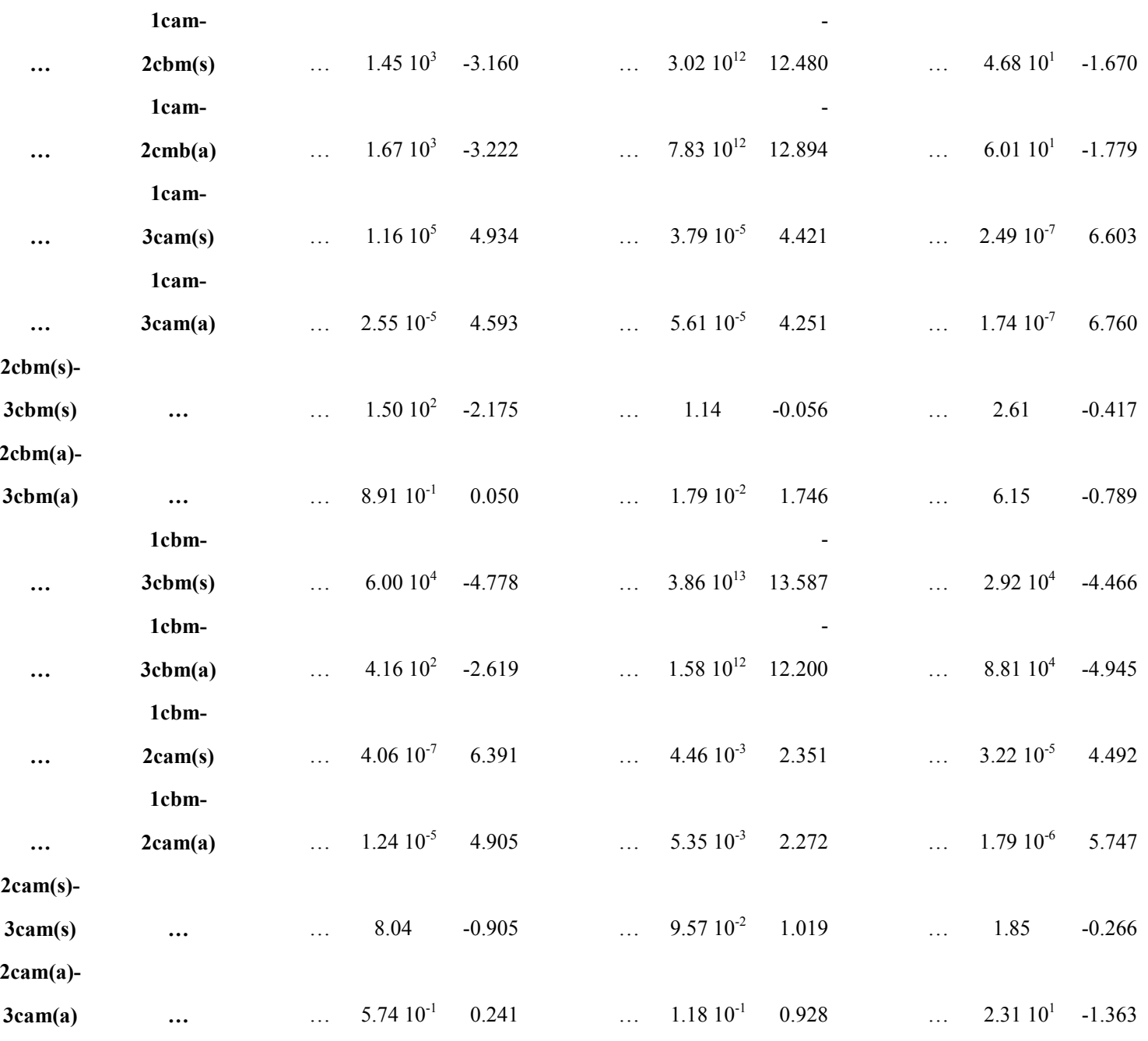




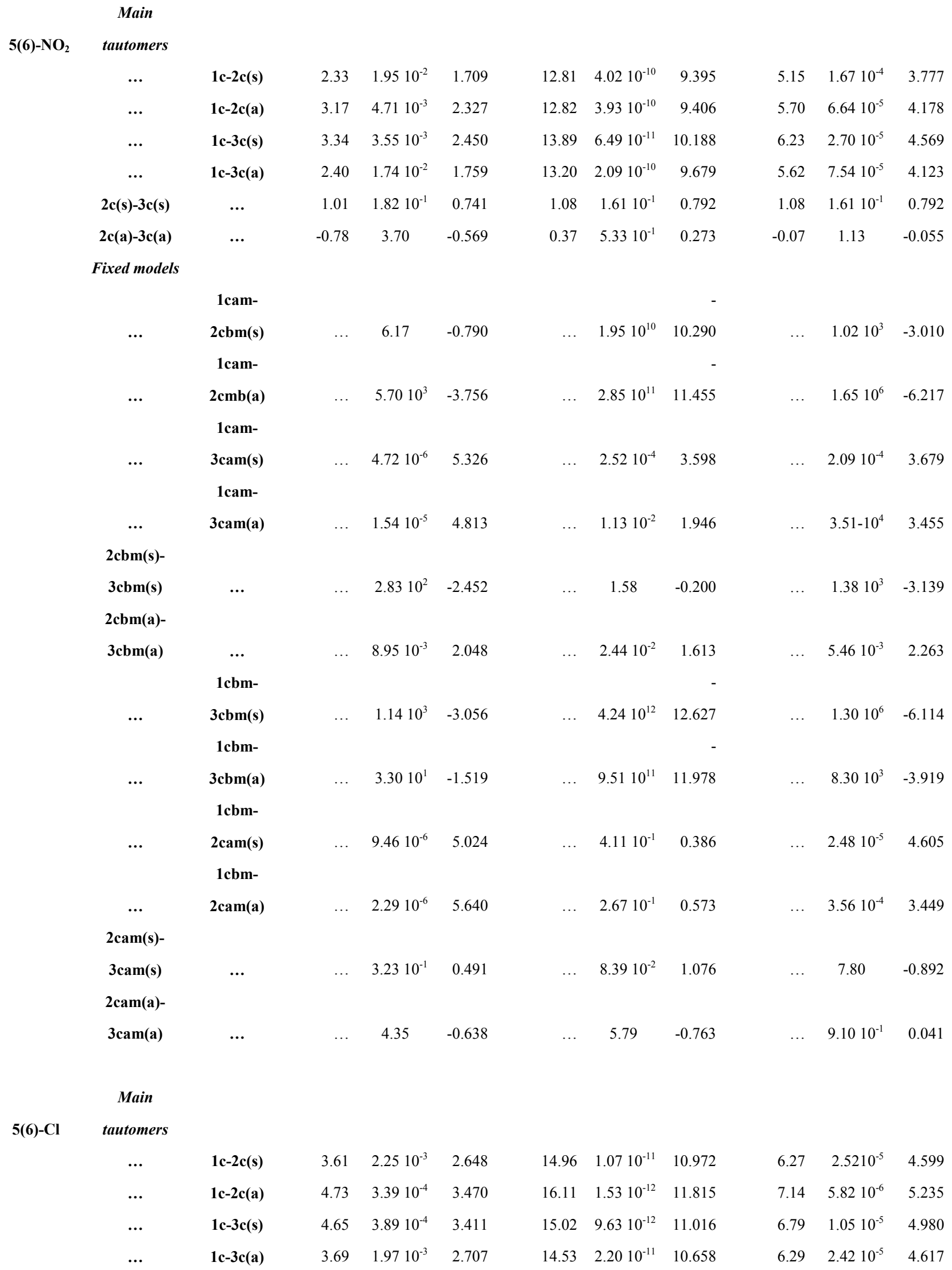




\begin{tabular}{|c|c|c|c|c|c|c|c|c|c|c|}
\hline $2 c(s)-3 c(s)$ & $\cdots$ & 1.04 & $1.7310^{-1}$ & 0.763 & 0.06 & $9.0410^{-1}$ & 0.044 & 0.52 & $4.1610^{-1}$ & 0.381 \\
\hline $2 c(a)-3 c(a)$ & $\cdots$ & -1.04 & 5.80 & -0.764 & -1.58 & $1.4310^{1}$ & -1.157 & -0.84 & 4.15 & -0.619 \\
\hline \multicolumn{11}{|l|}{ Fixed models } \\
\hline & 1cam- & & & & & & - & & & \\
\hline$\cdots$ & $2 \operatorname{cbm}(s)$ & $\ldots$ & $1.3810^{4}$ & -4.140 & $\ldots$ & $2.0010^{11}$ & 11.300 & $\cdots$ & $3.8010^{3}$ & -3.580 \\
\hline & 1cam- & & & & & & - & & & \\
\hline$\cdots$ & $2 \mathrm{cmb}(\mathrm{a})$ & $\ldots$ & $4.0010^{5}$ & -5.602 & $\cdots$ & $1.2010^{12}$ & 12.079 & $\ldots$ & $1.8110^{5}$ & -5.258 \\
\hline & 1cam- & & & & & & & & & \\
\hline$\cdots$ & $3 \operatorname{cam}(s)$ & $\ldots$ & $3.9210^{-4}$ & 3.407 & $\cdots$ & $1.7310^{-4}$ & 3.761 & $\cdots$ & $1.1410^{-4}$ & 3.944 \\
\hline$\cdots$ & $\begin{array}{c}\text { 1cam- } \\
3 \operatorname{cam}(a)\end{array}$ & $\cdots$ & $6.5910^{-4}$ & 3.181 & $\cdots$ & $5.8210^{-6}$ & 5.235 & $\cdots$ & $4.4310^{-5}$ & 4.354 \\
\hline \multicolumn{11}{|l|}{$2 \operatorname{cbm}(s)-$} \\
\hline $3 \operatorname{cbm}(s)$ & $\ldots$ & $\ldots$ & $1.1710^{2}$ & -2.068 & $\ldots$ & $5.3210^{-1}$ & 0.274 & $\ldots$ & 3.26 & -0.513 \\
\hline \multicolumn{11}{|l|}{$2 \operatorname{cbm}(a)-$} \\
\hline $3 \operatorname{cbm}(a)$ & $\cdots$ & $\ldots$ & $8.3210^{-1}$ & 0.080 & $\ldots$ & $1.7310^{-2}$ & 1.762 & $\ldots$ & $4.9010^{-3}$ & 2.310 \\
\hline & $1 \mathrm{cbm}-$ & & & & & & - & & & \\
\hline$\cdots$ & $3 \operatorname{cbm}(s)$ & $\ldots$ & $1.3610^{4}$ & -4.134 & $\ldots$ & $2.6310^{12}$ & 12.420 & $\ldots$ & $1.5110^{4}$ & -4.179 \\
\hline & $1 \mathrm{cbm}-$ & & & & & & - & & & \\
\hline$\cdots$ & $3 \operatorname{cbm}(a)$ & $\ldots$ & $2.8010^{3}$ & -3.447 & $\cdots$ & $5.1310^{11}$ & 11.710 & $\ldots$ & $1.0810^{3}$ & -3.034 \\
\hline & 1cbm- & & & & & & & & & \\
\hline$\cdots$ & 2 cam(s) & $\ldots$ & $7.6410^{-6}$ & 5.117 & $\ldots$ & $3.0010^{-5}$ & 4.523 & $\ldots$ & $5.3610^{-5}$ & 4.271 \\
\hline & 1cbm- & & & & & & & & & \\
\hline$\cdots$ & $2 \operatorname{cam}(a)$ & $\cdots$ & $1.9410^{-5}$ & 4.712 & $\cdots$ & $5.6910^{-4}$ & 3.245 & $\cdots$ & $1.3810^{-4}$ & 3.859 \\
\hline \multicolumn{11}{|l|}{2 cam(s)- } \\
\hline $3 \operatorname{cam}(s)$ & $\cdots$ & $\cdots$ & $4.3110^{-1}$ & 0.366 & $\cdots$ & $1.4310^{2}$ & -2.155 & $\cdots$ & 2.59 & -0.413 \\
\hline \multicolumn{11}{|l|}{$2 \operatorname{cam}(a)-$} \\
\hline $3 \operatorname{cam}(a)$ & $\ldots$ & $\ldots$ & $2.8510^{-1}$ & 0.545 & $\ldots$ & $2.5410^{-1}$ & 0.596 & $\ldots$ & $3.9010^{-1}$ & 0.409 \\
\hline
\end{tabular}

${ }^{\mathrm{a}} \delta \Delta \mathrm{G}=\Delta \mathrm{G}_{\text {(product) }}-\Delta \mathrm{G}_{\text {(reactant) }}$

${ }^{\mathrm{b}} \mathrm{K}_{\mathrm{T}}=\mathrm{e}^{-(\delta \Delta \mathrm{G} / \mathrm{RT})}$ (for Main tautomers). $\mathrm{K}_{\mathrm{T}}=10^{-\mathrm{pK}}$ (for Fixed models).

${ }^{\mathrm{c}} \mathrm{pK} \mathrm{K}_{\mathrm{T}}=-\log \mathrm{K}_{\mathrm{T}}$ (for Main tautomer) $\mathrm{pK}_{\mathrm{T}}=\mathrm{pK}_{\mathrm{a} \text { (product) }}-\mathrm{pK}_{\mathrm{a} \text { (reactant) }}$ Charton's equaton [29]. The minus sign indicate a shift to right side of equilbrium (for Fixed models). 
Table 4. The aqueous phase PM3 ,PM5 and AM1 calculated some isomeric equilibrium constants $\mathrm{K}_{\mathrm{is}}$, of studied molecules

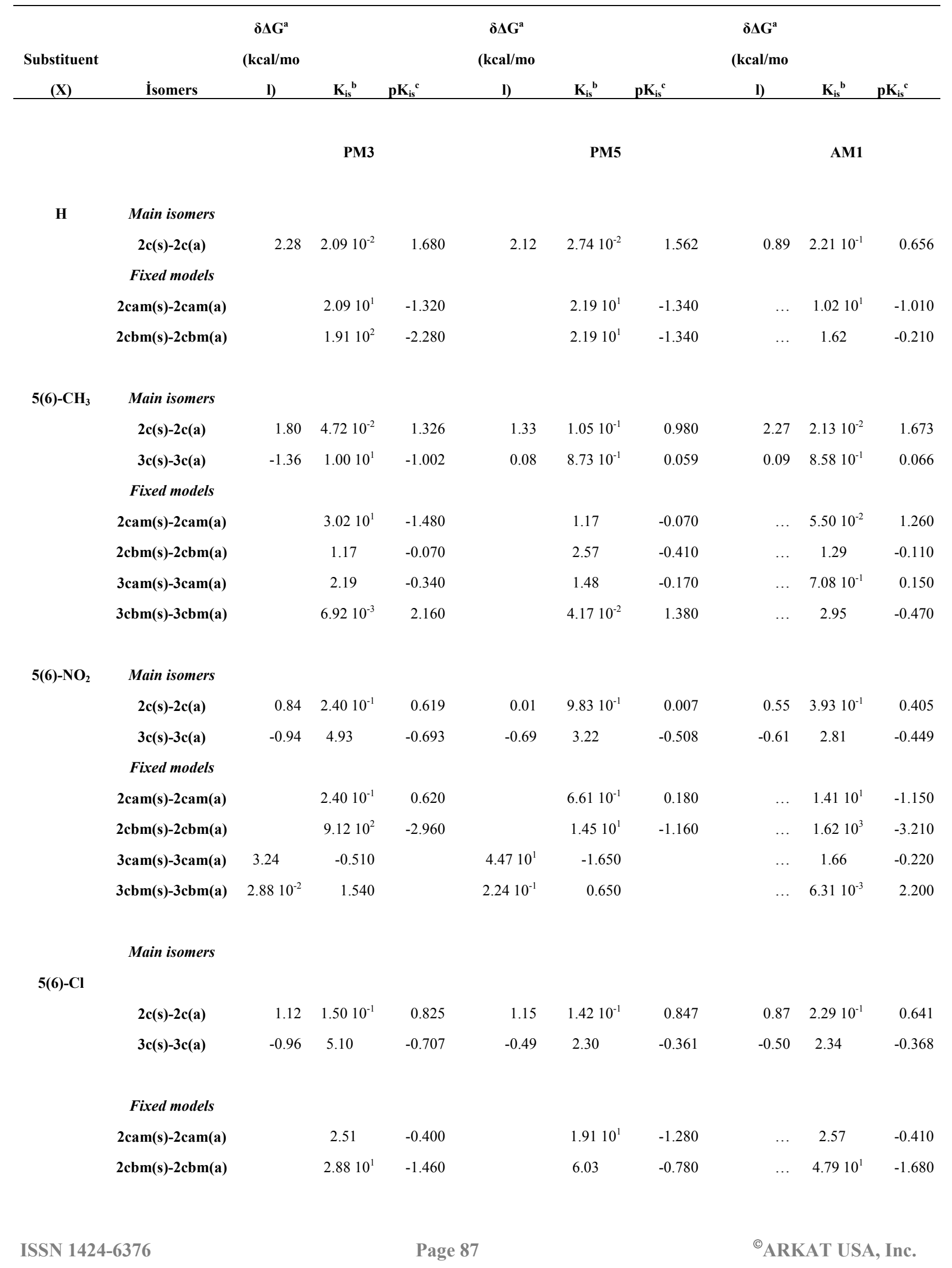




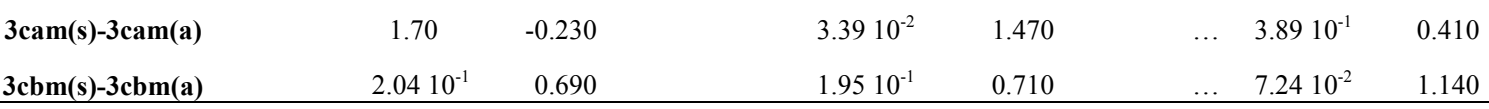

${ }^{\mathrm{a}} \delta \Delta \mathrm{G}=\Delta \mathrm{G}_{\text {(product) }}-\Delta \mathrm{G}_{\text {(reactant) }}$

${ }^{\mathrm{b}} \mathrm{K}_{\mathrm{is}}=\mathrm{e}^{-(\delta \Delta \mathrm{G} / \mathrm{RT})}$ (for main isomers). $\mathrm{K}_{\mathrm{T}}=10^{-\mathrm{pK}}{ }_{\mathrm{i}} \mathrm{s}$ (for half models).

${ }^{c} \mathrm{pK}_{\mathrm{is}}=-\log \mathrm{K}_{\mathrm{s}}$ (for main isomers) $\mathrm{pK}_{\mathrm{is}}=\mathrm{pK}_{\mathrm{a} \text { (product) }}-\mathrm{pK}_{\mathrm{a} \text { (reactant) }}$ Charton's equation [29]. The minus sign indicate a shift to right side of equilibrium (for Fixed models).

Table 5. The gas phase PM3, PM5 and AM1calculated relative stabilities for tautomers, RS, of studied molecules

\begin{tabular}{|c|c|c|c|c|c|}
\hline & & & nol) & & \\
\hline $\begin{array}{c}\text { Substiuent } \\
(\mathrm{X})\end{array}$ & $\begin{array}{c}\text { Annular } \\
\text { Tautomer }\end{array}$ & $\begin{array}{c}\text { Ring-chain } \\
\text { tautomer }\end{array}$ & PM3 & PM5 & AM1 \\
\hline \multirow[t]{8}{*}{ H } & Main tautomers & & & & \\
\hline & $\cdots$ & $1 c-2 c(s)$ & 2.87 & -10.97 & -3.65 \\
\hline & ... & $1 c-2 c(a)$ & 2.87 & -14.80 & -3.65 \\
\hline & Fixed models & & & & \\
\hline & $\ldots$ & $1 \mathrm{cam}-2 \operatorname{cam}(\mathrm{s})$ & 6.57 & -4.15 & 6.52 \\
\hline & ... & 1cam-2cam(a) & 6.57 & -7.41 & 3.68 \\
\hline & ... & $1 \mathrm{cam}-2 \operatorname{cbm}(s)$ & 2.53 & -11.19 & -3.64 \\
\hline & $\ldots$ & 1cam-2cbm(a) & -1.71 & -15.66 & -8.08 \\
\hline
\end{tabular}

5(6)- $\mathrm{CH}_{3} \quad$ Main tautomers

$\begin{array}{lcrrr}\ldots & \mathbf{1 c - 2 c ( s )} & 2.76 & -11.16 & -3.74 \\ \ldots & \mathbf{1 c - 2 c ( a )} & 2.76 & -15.00 & -3.74 \\ \ldots & \mathbf{1 c - 3 c ( s )} & -1.40 & -14.84 & -3.63 \\ \ldots & \mathbf{1 c - 3 c ( a )} & 2.82 & -11.02 & -3.63 \\ \mathbf{C}-\mathbf{3 c}(\mathbf{s}) & \ldots & -4.16 & -3.68 & 0.11 \\ \mathbf{3}-\mathbf{3 c}(\mathbf{a}) & \ldots & 0.06 & 3.98 & 0.11\end{array}$

Fixed

\begin{tabular}{|c|c|c|c|c|}
\hline ... & $1 \operatorname{cam}-2 \operatorname{cbm}(s)$ & -12.21 & -11.34 & -3.74 \\
\hline ... & $1 \mathrm{cam}-2 \mathrm{cmb}(\mathrm{a})$ & -16.45 & -15.85 & -8.17 \\
\hline ... & $1 \mathrm{cam}-3 \operatorname{cam}(\mathrm{s})$ & -12.23 & -7.47 & 3.70 \\
\hline ... & $1 \mathrm{cam}-3 \operatorname{cam}(a)$ & -8.11 & -4.22 & 6.52 \\
\hline $2 \operatorname{cbm}(s)-3 \operatorname{cbm}(s)$ & ... & -4.16 & -4.36 & -4.31 \\
\hline $2 \operatorname{cbm}(a)-3 \operatorname{cbm}(a)$ & ... & 4.29 & 4.63 & 4.54 \\
\hline ... & $1 \mathrm{cbm}-3 \operatorname{cbm}(s)$ & -1.74 & -15.69 & -8.04 \\
\hline ... & 1 cbm-3cbm(a) & 2.47 & -11.21 & -3.62 \\
\hline$\ldots$ & 1cbm-2cam(s) & 6.46 & -4.33 & 6.43 \\
\hline
\end{tabular}




\begin{tabular}{|c|c|c|c|c|c|}
\hline & $\ldots$ & $1 \mathrm{cbm}-2 \operatorname{cam}(a)$ & 2.33 & -7.60 & 3.59 \\
\hline & $2 \operatorname{cam}(s)-3 \operatorname{cam}(s)$ & $\ldots$ & -4.06 & -3.13 & -2.72 \\
\hline & $2 \operatorname{cam}(a)-3 \operatorname{cam}(a)$ & ... & 4.19 & 3.39 & 2.94 \\
\hline \multirow[t]{20}{*}{$5(6)-\mathrm{NO}_{2}$} & Main tautomers & & & & \\
\hline & $\ldots$ & $1 c-2 c(s)$ & 4.86 & -8.62 & -2.02 \\
\hline & ... & $1 c-2 c(a)$ & 0.29 & -12.50 & -5.53 \\
\hline & ... & $1 c-3 c(s)$ & 0.08 & -8.84 & -5.71 \\
\hline & ... & $1 c-3 c(a)$ & 4.63 & -8.86 & -2.30 \\
\hline & $2 c(s)-3 c(s)$ & ... & -4.78 & -0.22 & -3.69 \\
\hline & $2 c(a)-3 c(a)$ & ... & 4.34 & 3.64 & 3.23 \\
\hline & Fixed models & & & & \\
\hline & $\ldots$ & $1 \mathrm{cam}-2 \operatorname{cbm}(\mathrm{s})$ & 4.55 & -8.79 & -1.98 \\
\hline & $\ldots$ & 1 cam-2cmba & 0.19 & -13.39 & -6.60 \\
\hline & $\ldots$ & $1 \mathrm{cam}-3 \operatorname{cam}(\mathrm{s})$ & 4.21 & -1.85 & 5.23 \\
\hline & $\ldots$ & 1cam-3cam(a) & 8.46 & -1.87 & 8.09 \\
\hline & $2 \operatorname{cbm}(s)-3 \operatorname{cbm}(s)$ & ... & -4.59 & 0.01 & -4.63 \\
\hline & $2 \operatorname{cbm}(a)-3 \operatorname{cbm}(a)$ & $\ldots$ & 4.20 & 4.56 & 4.44 \\
\hline & $\ldots$ & $1 \mathrm{cbm}-3 \operatorname{cbm}(s)$ & -0.12 & -8.93 & -6.69 \\
\hline & $\ldots$ & $1 \mathrm{cbm}-3 \operatorname{cbm}(a)$ & 4.31 & -8.98 & -2.24 \\
\hline & $\ldots$ & $1 \mathrm{cbm}-2 \operatorname{cam}(s)$ & 8.63 & -1.78 & 8.27 \\
\hline & $\ldots$ & $1 \mathrm{cbm-2cam(a)}$ & 4.32 & -5.19 & 5.24 \\
\hline & $2 \operatorname{cam}(s)-3 \operatorname{cam}(s)$ & ... & -4.50 & -0.22 & -3.12 \\
\hline & $2 \operatorname{cam}(a)-3 \operatorname{cam}(a)$ & $\ldots$ & 4.06 & 3.17 & 2.77 \\
\hline
\end{tabular}

5(6)-Cl Main tautomers

$\ldots$
$\ldots$
$\ldots$
$\cdots$
$2 c(s)-3 c(s)$
$2 c(a)-3 c(a)$

$1 \mathrm{c}-2 \mathrm{c}(\mathrm{s})$
$1 \mathrm{c}-2 \mathrm{c}(\mathrm{a})$
$1 \mathrm{c}-3 \mathrm{c}(\mathrm{s})$
$1 \mathrm{c}-3 \mathrm{c}(\mathrm{a})$
$\ldots$
$\ldots$

$\begin{array}{rrr}3.30 & -10.28 & -3.20 \\ 3.30 & -14.16 & -6.64 \\ -0.91 & -13.96 & -6.48 \\ 3.40 & -10.15 & -3.11 \\ -4.21 & -3.68 & -3.28 \\ 0.10 & 4.01 & 3.53\end{array}$

Fixed models

Table 5 cont.

$$
\text { ... 1cam-2cbm(s) }
$$

$2.97 \quad-10.46$

$\begin{array}{cc}\ldots & 1 \mathrm{cam}-2 \mathrm{cmba} \\ \ldots & 1 \mathrm{cam}-3 \operatorname{cam}(\mathrm{s}) \\ \ldots & 1 \mathrm{cam}-3 \operatorname{cam}(\mathrm{a}) \\ 2 \operatorname{cbm}(\mathbf{s})-3 \operatorname{cbm}(\mathrm{s}) & \ldots \\ 2 \operatorname{cbm}(\mathrm{a})-3 \operatorname{cbm}(a) & \ldots\end{array}$

$\begin{array}{rrr}-1.33 & -15.02 & -7.68 \\ 3.00 & -6.53 & 4.28 \\ 7.14 & -3.29 & 7.11 \\ -4.13 & -4.33 & -4.32 \\ 4.41 & 4.72 & 4.60\end{array}$




\begin{tabular}{ccrrr}
$\ldots$ & $\mathbf{1 c b m - 3 c b m ( s )}$ & -1.18 & -14.82 & -7.52 \\
$\ldots$ & $\mathbf{1 c b m - 3 c b m ( a )}$ & 3.06 & -10.33 & -3.09 \\
$\ldots$ & $\mathbf{1 c b m}-2 \mathbf{c a m}(\mathbf{s})$ & 7.03 & -3.43 & 7.02 \\
$\ldots$ & $\mathbf{1 c b m}-2 \mathbf{c a m}(\mathbf{a})$ & 2.83 & -6.75 & 4.12 \\
$\mathbf{2 c a m}(\mathbf{s})-\mathbf{3 c a m}(\mathbf{s})$ & $\ldots$ & -4.05 & -3.13 & -2.75 \\
$\mathbf{2 c a m}(\mathbf{a})-\mathbf{3 c a m}(\mathbf{a})$ & $\ldots$ & 4.29 & 3.43 & 2.98 \\
\hline
\end{tabular}

${ }^{\mathrm{a}} \mathrm{RS}=\Delta \mathrm{H}_{\mathrm{f}(\text { reactant })}-\Delta \mathrm{H}_{\mathrm{f}(\text { product })}$

For 5(6)-substituted benzimidazole derivatives (i.e. molecules II-IV) the tautomeric equilibrium constants indicate that the thione form $\mathbf{1 c}$ is predominant over the thiol forms $\mathbf{2 c}$ and 3c with all methods. For the model compounds 5(6)-methylbenzimidazole III and 5(6)chlorobenzimidazole $\mathrm{V}$ derivatives the tautomeric equilibrium constants indicate the predominance of thiol forms $\mathbf{2} \mathbf{c a m}$ and $\mathbf{2 c b m}$ over the thione forms $\mathbf{1 c a m}$ and $\mathbf{1 c b m}$ with all methods. For the model compounds of 5(6)-nitro benzimidazole IV, the tautomeric equilibrium constants indicate that thiol form $\mathbf{2} \mathbf{c a m}$ and $\mathbf{2} \mathbf{c b m}$ is predominant over the thione form $\mathbf{1} \mathbf{c a m}$ and 1cbm with AM1, PM3 and PM5 methods.

When a potential mercapto group is located at C-2 for benzimidazole, the relative stability data indicate that the thione form I 1b predominates over the thiol form I 2c AM1 and PM5 calculations, whereas PM3 data indicate the reverse. PM5 calculation for the model compounds suggest that the predominance of thione form $\mathbf{1 b m}$ over the thiol forms $\mathbf{2} \mathbf{c a m}$ and $\mathbf{2} \mathbf{c b m}$. However the AM1 and PM3 methods suggest the reverse. For 5(6)-substituted benzimidazole derivatives (substituents; $\mathrm{CH}_{3}, \mathrm{NO}_{2}$ and $\mathrm{Cl}$ ) the relative stability data of AM1 and PM5 methods indicate that thione form $\mathbf{1 c}$ is predominant over the thiol forms $\mathbf{2 c}$ and $\mathbf{3 c}$. However, the PM3 method indicates the reverse. For the model compounds of 5(6)-methylbenzimidazole the relative stability data indicate the predominance of thione forms $\mathbf{1 c a m}$ and $\mathbf{1} \mathbf{c b m}$ over the thiol forms $\mathbf{2 c a m}$ and $\mathbf{2} \mathbf{c b m}$ and $\mathbf{3 c a m}$ and $\mathbf{3 c b m}$ with AM1, PM3 and PM5 methods. Similarly for the model compounds of 5(6)-nitrobenzimidazole and 5(6)-chlorobenzimidazole the relative stability data indicate that the 3 thione forms $\mathbf{1}$ cam and $\mathbf{1} \mathbf{c b m}$ are predominant over the thiol forms $\mathbf{2 c a m}$ and $\mathbf{2 c b m}$ and $\mathbf{3 c a m}$ and $\mathbf{3 c b m}$ with AM1 and PM5 methods. Whereas with PM3 methods the reverse is true. In the literature, the thione structure was shown to be the stable form of benzimidazole-2-thione derivatives. ${ }^{31-33}$

\section{Acidity}

The protonation reaction of a given base can be shown as follows;

$$
\begin{gathered}
\mathrm{BH}^{\mathrm{n}+} \rightleftharpoons \mathrm{B}^{(\mathrm{n}+1)+}+\mathrm{H}^{+} \\
\delta \Delta \mathrm{G}_{(\mathrm{BH}+)}=\left[\Delta \mathrm{G}_{(\mathrm{B})}+\Delta \mathrm{G}_{(\mathrm{AH}+)}\right]-\left[\Delta \mathrm{G}_{(\mathrm{BH}+)}+\Delta \mathrm{G}_{(\mathrm{A})}\right]
\end{gathered}
$$

in which $\mathrm{n}$ can have a negative, positive or zero value. The acidity of a given base, $\mathrm{B}$, for the protonation reaction can be calculated using Eq.2. in which $\mathrm{B}$ and $\mathrm{BH}^{+}$are neutral and protonated species of base $\mathrm{B}$, and $\mathrm{A}$ and $\mathrm{AH}^{+}$are $\mathrm{H}_{2} \mathrm{O}$ and $\mathrm{H}_{3} \mathrm{O}^{+}$respectively. The computed 
thermodynamic data were used in predicting the acidity constants, $\mathrm{pK}_{\mathrm{a}}$ values, of various species, using Eq. 3, in which the $\delta \Delta \mathrm{G}_{(\mathrm{BH}+)}$ is the standard free energy change for the protonation reaction (Eq. 1) (Table 6).

$$
\mathrm{pK}_{\mathrm{a}(\mathrm{BH}+)}=\delta \Delta \mathrm{G}_{(\mathrm{BH}+)} / 2.303 \mathrm{RT}
$$

Possible deprotonation patterns for the studied molecules are shown in Schemes 1 and 2. Aqueous phase calculated acidity constant $\mathrm{pK}_{\mathrm{a}}$ values are given in Table 3 . From these data the deprotonation $\mathrm{pK}_{\mathrm{a}}$ values of 5(6)-methylbenzimidazole-2-thiol was found to be the highest (i.e. 13.41 by the AM1method) and the deprotonation $\mathrm{pK}_{\mathrm{a}}$ values of 5(6)-nitrobenzimidazole-2-thiol was found to be the lowest (i.e. 11.71 by the AM1 method).

Experimental $\mathrm{pK}_{\mathrm{a}}: \mathrm{CH}_{3^{-}}>\mathrm{H}->\mathrm{Cl}->\mathrm{NO}_{2^{-}}$

$$
11.65>10.24>9.06>7.98
$$

AM1 method $\mathrm{pK}_{\mathrm{a}}: \mathrm{CH}_{3^{-}}>\mathrm{H}->\mathrm{Cl}->\mathrm{NO}_{2^{-}}$

$13.41>12.94>12.51>11.71$

decreasing acidity

We have attempted to correlate the experimental $\mathrm{pK}_{\mathrm{a}}$ values ${ }^{34,35}$ with calculated $\mathrm{pK}_{\mathrm{a}}$ values and we observed that the best fit occurred with the AM1 method (Fig. 1).

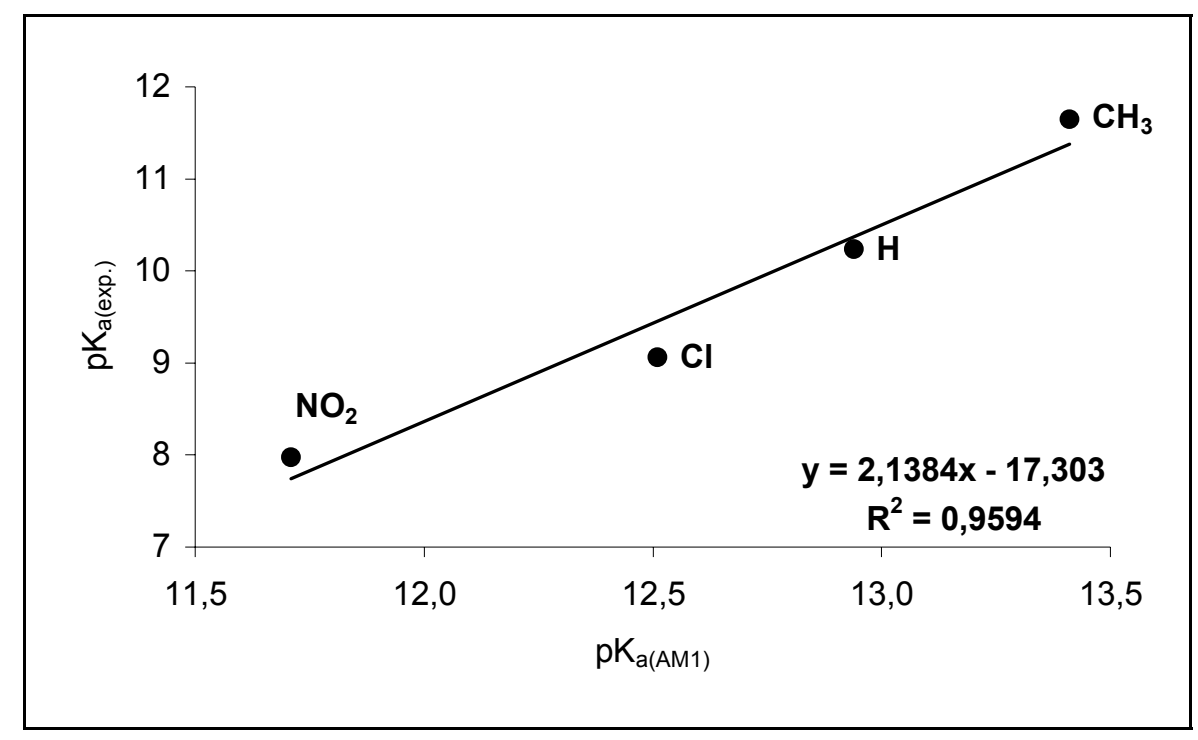

Figure 1. Plot of the aqueous phase $A M 1$ calculated acidity constants, $\mathrm{pK}_{\mathrm{a}}$ (calc.), and experimental acidity constants, $\mathrm{pK}_{\mathrm{a}}$ (exp.), for the studied molecules. 
Table 6. Aqueous phase PM3, PM5 and AM1 calculated $\mathrm{pK}_{\mathrm{a}}$ values for the investigated molecules

\begin{tabular}{|c|c|c|c|c|c|c|c|c|c|}
\hline & & & PM3 & & PM5 & & AM1 & & \\
\hline $\begin{array}{c}\text { Substituent } \\
(\mathrm{X}) \\
\end{array}$ & $\begin{array}{c}\text { Anion } \\
\left(\mathrm{B}^{-}\right) \\
\end{array}$ & Neutral (BH) & $\begin{array}{c}\delta \Delta \mathbf{G}_{(\mathrm{BH}+)} \\
(\mathrm{kcal} / \mathrm{mol})^{\mathrm{a}} \\
\end{array}$ & $\mathbf{p K a}^{\mathbf{b}}$ & $\begin{array}{c}\delta \Delta \mathbf{G}_{(\mathrm{BH}+)} \\
(\mathrm{kcal} / \mathrm{mol})^{\mathrm{a}} \\
\end{array}$ & $\mathbf{p K a}^{\mathbf{b}}$ & $\begin{array}{c}\delta \Delta \mathbf{G}_{(\mathrm{BH}+)} \\
(\mathrm{kcal} / \mathbf{m o l})^{\mathrm{a}}\end{array}$ & $\mathbf{p K a}^{\mathbf{b}}$ & pKa(exp.) $)^{\mathrm{c}}$ \\
\hline \multirow[t]{10}{*}{$\mathbf{H}$} & $1 \mathrm{a}$ & $1 \mathrm{~b}$ & 30.07 & 22.05 & 6.70 & 4.91 & 24.99 & 18.33 & 10.24 \\
\hline & $2 a(s)$ & $2 \mathrm{c}(\mathrm{s})$ & 36.45 & 26.73 & 14.00 & 10.27 & 31.09 & 22.80 & \\
\hline & $2 a(a)$ & $2 c(a)$ & 34.17 & 25.06 & 11.87 & 8.71 & 30.32 & 22.23 & \\
\hline & $2 \mathbf{b}$ & $2 c(s)$ & 26.31 & 19.29 & -8.45 & -6.20 & 18.53 & 13.59 & \\
\hline & $2 \mathbf{b}$ & $2 c(a)$ & 24.03 & 17.62 & -10.57 & -7.75 & 17.64 & 12.94 & \\
\hline & $1 \mathrm{am}$ & $1 \mathrm{bm}$ & 30.11 & 22.08 & 6.40 & 4.69 & 27.32 & 20.03 & \\
\hline & $2 \mathrm{am}(\mathrm{s})$ & $2 \operatorname{cam}(s)$ & 38.59 & 28.30 & 14.60 & 10.71 & 31.90 & 23.39 & \\
\hline & 2am(a) & $2 \operatorname{cam}(a)$ & 35.49 & 26.02 & 12.77 & 9.37 & 31.61 & 23.18 & \\
\hline & $2 \mathrm{bm}$ & $2 \operatorname{cbm}(s)$ & 24.68 & 18.10 & -10.76 & -7.89 & 18.36 & 13.46 & \\
\hline & $2 \mathrm{bm}$ & $2 \operatorname{cbm}(a)$ & 22.88 & 16.78 & -12.58 & -9.23 & 16.98 & 12.45 & \\
\hline \multirow[t]{20}{*}{$5(6)-\mathrm{CH}_{3}$} & $1 \mathrm{a}$ & $1 \mathrm{c}$ & 32.08 & 23.52 & 8.56 & 6.28 & 25.48 & 18.68 & 11.65 \\
\hline & $1 \mathrm{~b}$ & $1 \mathrm{c}$ & 30.75 & 22.55 & 7.14 & 5.24 & 23.78 & 17.44 & \\
\hline & $2 \mathrm{a}(\mathrm{s})$ & $2 \mathrm{c}(\mathrm{s})$ & 36.30 & 26.62 & 13.68 & 10.03 & 32.51 & 23.84 & \\
\hline & $2 a(a)$ & $2 c(a)$ & 34.71 & 25.46 & 12.35 & 9.06 & 28.70 & 21.05 & \\
\hline & $2 \mathrm{~b}$ & $2 c(s)$ & 26.44 & 19.39 & -8.47 & -6.21 & 18.28 & 13.41 & \\
\hline & $2 b$ & $2 c(a)$ & 24.64 & 18.07 & -9.80 & -7.18 & 16.01 & 11.74 & \\
\hline & $3 a(s)$ & $3 c(s)$ & 34.98 & 25.65 & 12.59 & 9.23 & 30.60 & 22.44 & \\
\hline & $3 a(a)$ & $3 \mathbf{c}(\mathbf{a})$ & 36.68 & 26.90 & 12.48 & 9.15 & 29.64 & 21.74 & \\
\hline & $3 b$ & $3 c(s)$ & 25.46 & 18.67 & -9.27 & -6.80 & 17.53 & 12.86 & \\
\hline & $3 b$ & $3 \mathbf{c}(\mathbf{a})$ & 26.83 & 19.67 & -9.35 & -6.85 & 17.44 & 12.79 & \\
\hline & $1 \mathrm{am}$ & $1 \mathrm{cam}$ & 30.73 & 22.53 & 7.43 & 5.45 & 22.33 & 16.37 & \\
\hline & $1 \mathrm{bm}$ & $1 \mathrm{cbm}$ & 29.98 & 21.98 & 8.87 & 6.50 & 25.57 & 18.75 & \\
\hline & $2 \mathrm{am}(\mathrm{s})$ & $2 \operatorname{cam}(\mathrm{s})$ & 38.69 & 28.37 & 12.07 & 8.85 & 31.70 & 23.24 & \\
\hline & 2am(a) & 2cam(a) & 36.66 & 26.89 & 11.97 & 8.78 & 33.41 & 24.50 & \\
\hline & $2 \mathrm{bm}$ & $2 \operatorname{cbm}(s)$ & 26.43 & 19.38 & -9.58 & -7.03 & 20.05 & 14.70 & \\
\hline & $2 \mathrm{bm}$ & $2 \operatorname{cbm}(a)$ & 26.34 & 19.31 & -10.15 & -7.44 & 19.90 & 14.59 & \\
\hline & 3am(s) & $3 \operatorname{cam}(s)$ & 37.46 & 27.47 & 13.46 & 9.87 & 31.33 & 22.98 & \\
\hline & 3am(a) & $3 \operatorname{cam}(a)$ & 36.99 & 27.13 & 13.23 & 9.70 & 31.55 & 23.13 & \\
\hline & $3 \mathrm{bm}$ & $3 \operatorname{cbm}(s)$ & 23.46 & 17.20 & -9.66 & -7.08 & 19.48 & 14.28 & \\
\hline & $3 \mathrm{bm}$ & $3 \operatorname{cbm}(a)$ & 26.40 & 19.36 & -7.77 & -5.70 & 18.83 & 13.81 & \\
\hline \multirow[t]{3}{*}{$5(6)-\mathrm{NO}_{2}$} & $1 \mathrm{a}$ & $1 \mathrm{c}$ & 26.15 & 19.18 & 3.00 & 2.20 & 22.27 & 16.33 & 7.98 \\
\hline & $1 \mathrm{~b}$ & $1 \mathrm{c}$ & 25.13 & 18.43 & 3.03 & 2.22 & 22.18 & 16.27 & \\
\hline & $2 a(s)$ & $2 \mathrm{c}(\mathrm{s})$ & 31.95 & 23.43 & 6.84 & 5.02 & 26.77 & 19.63 & \\
\hline
\end{tabular}




\begin{tabular}{|c|c|c|c|c|c|c|c|c|c|}
\hline & $2 a(a)$ & $2 \mathrm{c}(\mathrm{a})$ & 31.29 & 22.95 & 6.83 & 5.01 & 26.13 & 19.16 & \\
\hline & $2 b$ & $2 \mathrm{c}(\mathrm{s})$ & 23.83 & 17.47 & -9.82 & -7.20 & 17.12 & 12.55 & \\
\hline & $2 b$ & $2 c(a)$ & 22.99 & 16.86 & -9.84 & -7.21 & 16.57 & 12.15 & \\
\hline & $3 a(s)$ & $3 c(s)$ & 30.95 & 22.70 & 5.75 & 4.22 & 25.69 & 18.84 & \\
\hline & $3 a(a)$ & $3 \mathrm{c}(\mathrm{a})$ & 32.07 & 23.51 & 6.45 & 4.73 & 26.21 & 19.22 & \\
\hline & $3 \mathbf{b}$ & $3 c(s)$ & 24.40 & 17.89 & -12.68 & -9.30 & 15.97 & 11.71 & \\
\hline & $3 b$ & $3 c(a)$ & 25.34 & 18.58 & -11.99 & -8.79 & 16.58 & 12.16 & \\
\hline & $1 \mathrm{am}$ & 1cam & 25.50 & 18.70 & 3.25 & 2.38 & 22.24 & 16.31 & \\
\hline & $1 \mathrm{bm}$ & $1 \mathrm{cbm}$ & 25.24 & 18.51 & 6.16 & 4.52 & 22.20 & 16.28 & \\
\hline & $2 a m(s)$ & $2 \operatorname{cam}(s)$ & 32.09 & 23.53 & 6.69 & 4.91 & 28.48 & 20.88 & \\
\hline & 2am(a) & $2 \operatorname{cam}(a)$ & 32.93 & 24.15 & 6.95 & 5.09 & 26.90 & 19.73 & \\
\hline & $2 \mathrm{bm}$ & $2 \operatorname{cbm}(s)$ & 24.42 & 17.90 & -10.78 & -7.91 & 18.14 & 13.30 & \\
\hline & $2 \mathrm{bm}$ & $2 \operatorname{cbm}(a)$ & 20.37 & 14.94 & -12.37 & -9.07 & 13.77 & 10.09 & \\
\hline & 3am(s) & $3 \operatorname{cam}(s)$ & 32.76 & 24.02 & 8.16 & 5.98 & 27.26 & 19.99 & \\
\hline & 3am(a) & $3 \operatorname{cam}(a)$ & 32.06 & 23.51 & 5.91 & 4.33 & 26.96 & 19.77 & \\
\hline & $3 \mathrm{bm}$ & $3 \operatorname{cbm}(s)$ & 21.07 & 15.45 & -11.05 & -8.11 & 13.86 & 10.16 & \\
\hline & $3 \mathrm{bm}$ & $3 \operatorname{cbm}(a)$ & 23.17 & 16.99 & -10.17 & -7.46 & 16.85 & 12.36 & \\
\hline \multirow[t]{20}{*}{ 5(6)-Cl } & $1 \mathrm{a}$ & $1 \mathrm{c}$ & 29.68 & 21.76 & 5.80 & 4.25 & 24.20 & 17.75 & 9.06 \\
\hline & $1 \mathrm{~b}$ & $1 \mathrm{c}$ & 29.64 & 21.73 & 5.92 & 4.34 & 24.25 & 17.78 & \\
\hline & $2 a(s)$ & $2 \mathrm{c}(\mathrm{s})$ & 35.28 & 25.87 & 12.17 & 8.92 & 29.49 & 21.62 & \\
\hline & 2a(a) & $2 c(a)$ & 34.24 & 25.11 & 11.02 & 8.08 & 28.60 & 20.98 & \\
\hline & $2 \mathrm{~b}$ & $2 \mathrm{c}(\mathrm{s})$ & 26.07 & 19.12 & -9.16 & -6.72 & 17.93 & 13.15 & \\
\hline & $2 b$ & $2 c(a)$ & 24.95 & 18.29 & -10.31 & -7.56 & 17.06 & 12.51 & \\
\hline & $3 a(s)$ & $3 \mathrm{c}(\mathrm{s})$ & 34.24 & 25.11 & 12.11 & 8.88 & 28.97 & 21.24 & \\
\hline & 3a(a) & $3 \mathbf{c}(\mathbf{a})$ & 35.27 & 25.87 & 12.60 & 9.24 & 29.42 & 21.58 & \\
\hline & $3 b$ & $3 c(s)$ & 24.99 & 18.32 & -9.09 & -6.67 & 17.46 & 12.80 & \\
\hline & $3 b$ & $3 c(a)$ & 25.95 & 19.03 & -8.61 & -6.31 & 17.95 & 13.16 & \\
\hline & $1 \mathrm{am}$ & 1cam & 31.37 & 23.00 & 5.29 & 3.88 & 24.28 & 17.81 & \\
\hline & $1 \mathrm{bm}$ & $1 \mathrm{cbm}$ & 28.54 & 20.93 & 7.19 & 5.27 & 24.40 & 17.89 & \\
\hline & $2 \mathrm{am}(\mathrm{s})$ & 2cam(s) & 35.51 & 26.04 & 13.36 & 9.80 & 30.23 & 22.16 & \\
\hline & 2am(a) & 2cam(a) & 34.96 & 25.64 & 11.62 & 8.52 & 29.66 & 21.75 & \\
\hline & $2 \mathrm{bm}$ & $2 \operatorname{cbm}(s)$ & 25.72 & 18.86 & -10.12 & -7.42 & 19.40 & 14.23 & \\
\hline & $2 \mathrm{bm}$ & $2 \operatorname{cbm}(a)$ & 23.73 & 17.40 & -11.18 & -8.20 & 17.11 & 12.55 & \\
\hline & 3am(s) & $3 \operatorname{cam}(s)$ & 36.01 & 26.41 & 10.42 & 7.64 & 29.66 & 21.75 & \\
\hline & 3am(a) & $3 \operatorname{cam}(a)$ & 35.70 & 26.18 & 12.43 & 9.11 & 30.22 & 22.16 & \\
\hline & $3 b m$ & $3 \operatorname{cbm}(s)$ & 22.90 & 16.79 & -9.75 & -7.15 & 18.70 & 13.72 & \\
\hline & $3 \mathrm{bm}$ & $3 \operatorname{cbm}(a)$ & 23.83 & 17.48 & -8.78 & -6.44 & 20.26 & 14.86 & \\
\hline
\end{tabular}

${ }^{\mathrm{a}} \delta \Delta \mathrm{G}_{(\mathrm{BH}+)}=\left[\Delta \mathrm{G}_{(\mathrm{B})}+\Delta \mathrm{G}_{(\mathrm{H} 3 \mathrm{O}+)}\right]-\left[\Delta \mathrm{G}_{(\mathrm{BH})}+\Delta \mathrm{G}_{(\mathrm{H} 2 \mathrm{O})}\right]$

${ }^{\mathrm{b}} \mathrm{pK}_{\mathrm{a}}=\delta \Delta \mathrm{G}_{(\mathrm{BH})} /(2.303 \mathrm{RT})$

${ }^{\mathrm{c}} \mathrm{pK}_{\mathrm{a}}$ (expt.) were taken from ref.[6] 


\section{Nucleophilicity Criteria}

The principle of hard and soft bases has been applied to kinetic phenomena for a long time. In this connection, organic chemistry has provided most of the examples, because organic reactions are often slow enough for their rates to be easily measured. In organic chemistry, we are generally interested in the reactions of electrophiles and nucleophiles. These reactions are a particular kind of the general acid-with-base type of reaction, and so the principle of hard and soft acids and bases applies equally to the reactions of electrophiles and nucleophiles. So acidity and basicity can be related to the theoretical interpretation of hard-soft acids and bases stating the following principles:

i. Hard acids have high-energy LUMOs and the hard bases have low-energy HOMOs.

ii. The lower the energy of the HOMO of a base the harder it is as a base.

iii. A hard acid bonds strongly to a hard base because the orbitals involved are far apart in energy.

iv. A soft acid bonds strongly to a soft base because the orbitals involved are close in energy.

Since it was observed that the rates with which nucleophiles attack one kind of electrophile are not necessarily a good guide to the rates with which the same nucleophile will attack other electrophiles, so it is a good idea to categorize nucleophiles as being hard or soft, and electrophiles as being soft or hard. The theoretical interpretation of them are as follows;

i. Hard nucleophiles have relatively low-energy HOMOs.

ii. Soft nucleophiles have high- energy HOMOs.

The solvated proton is a hard electrophile and little affected by frontier orbital interactions. For this reason, the $\mathrm{pK}_{\mathrm{a}}$ of the conjugated acid of a nucleophile is a good measure of the rate at which that a nucleophile will attack another hard electrophile. ${ }^{36}$

Taking all those above mentioned points into account, we have attempted to find possible correlations between the experimental or computed acidity constants, $\mathrm{pK}_{\mathrm{a}}$ values, and computed nucleophilicity of the studied molecules.

The nucleophilicity, $\mathrm{n}=\mathrm{E}_{\mathrm{HOMO}}-\mathrm{E}_{\mathrm{LUMO}}$ [37], of the studied molecules was calculated and the aqueous phase calculated $n$ values of the studied neutral molecules are depicted in Table 7.

It seems that the basicity and nucleophilicity of compound IV is reduced as expected from the strong electron-withdrawing effect of the nitro group. Whereas the weaker electronwithdrawing effect of chlorine reduces the basicity and nucleophilicity to a lower extent. On the other hand, the electron-donating methyl group seems to increase the basicity power of molecule II but not its nucleophilicity (Figs. 2-3) 
Table 7. The aqueous phase PM3, PM5 and AM1calculated nucleophilicity (n) values for studied molecules

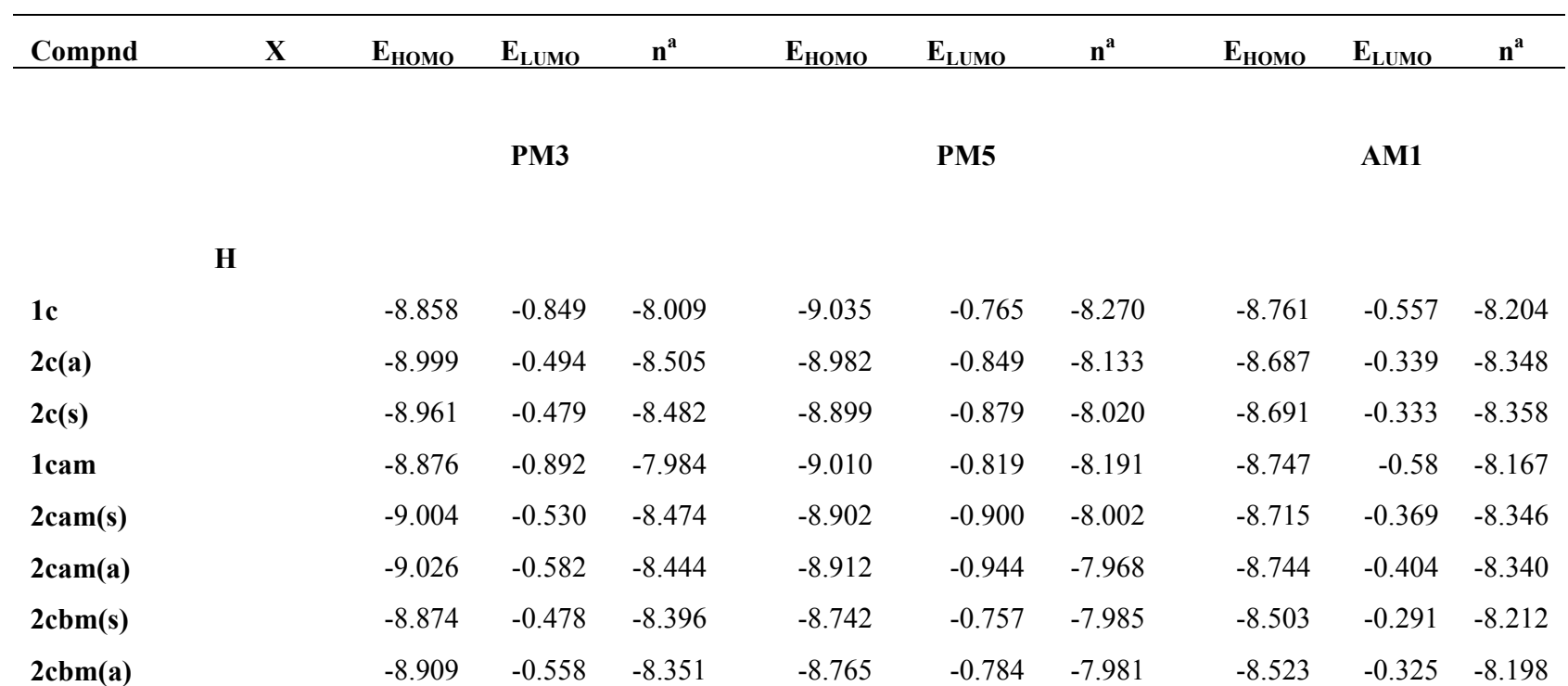

$5(6)-\mathrm{CH}_{3}$

1c

$2 c(a)$

$2 \mathrm{c}(\mathrm{s})$

$3 \mathbf{c}(\mathbf{a})$

$3 c(s)$

1 cam

$1 \mathrm{cbm}$

$2 \mathrm{cam}(\mathrm{s})$

$2 \operatorname{cam}(a)$

$2 \operatorname{cbm}(s)$

$2 \operatorname{cbm}(\mathbf{a})$

$3 \operatorname{cam}(s)$

$3 \operatorname{cam}(\mathbf{a})$

$3 \operatorname{cbm}(s)$

$3 \operatorname{cbm}(a)$

$\begin{array}{rrrrrrrrr}-8.815 & -0.842 & -7.973 & -8.938 & -0.765 & -8.173 & -8.700 & -0.560 & -8.140 \\ -8.965 & -0.488 & -8.477 & -8.868 & -0.848 & -8.020 & -8.648 & -0.332 & -8.316 \\ -8.914 & -0.473 & -8.441 & -8.803 & -0.873 & -7.930 & -8.648 & -0.324 & -8.324 \\ -8.859 & -0.474 & -8.385 & -8.762 & -0.872 & -7.890 & -8.601 & -0.347 & -8.254 \\ -8.854 & -0.498 & -8.356 & -8.770 & -0.866 & -7.904 & -8.598 & -0.355 & -8.243 \\ -8.834 & -0.885 & -7.949 & -8.907 & -0.815 & -8.092 & -8.683 & -0.579 & -8.104 \\ -8.834 & -0.887 & -7.947 & -8.921 & -0.815 & -8.106 & -8.687 & -0.582 & -8.105 \\ -8.830 & -0.472 & -8.358 & -8.668 & -0.753 & -7.915 & -8.471 & -0.282 & -8.189 \\ -8.879 & -0.553 & -8.326 & -8.701 & -0.781 & -7.920 & -8.496 & -0.316 & -8.180 \\ -8.951 & -0.524 & -8.427 & -8.792 & -0.896 & -7.896 & -8.670 & -0.360 & -8.310 \\ -8.977 & -0.578 & -8.399 & -8.929 & -0.853 & -8.076 & -8.703 & -0.396 & -8.307 \\ -8.818 & -0.552 & -8.266 & -8.651 & -0.778 & -7.873 & -8.452 & -0.339 & -8.113 \\ -8.789 & -0.472 & -8.317 & -8.635 & -0.752 & -7.883 & -8.434 & -0.305 & -8.129 \\ -8.918 & -0.577 & -8.341 & -8.998 & -0.825 & -8.173 & -8.654 & -0.420 & -8.234 \\ -8.897 & -0.523 & -8.374 & -8.771 & -0.895 & -7.876 & -8.621 & -0.383 & -8.238\end{array}$

5(6)-NO

$1 c$

$2 c(a)$

$2 \mathrm{c}(\mathrm{s})$

$3 c(a)$

$3 c(s)$

1 cam

$\begin{array}{lll}-8.990 & -1.429 & -7.561 \\ -9.146 & -1.132 & -8.014 \\ -9.154 & -1.140 & -8.014 \\ -9.204 & -1.235 & -7.969 \\ -9.199 & -1.243 & -7.956 \\ -9.011 & -1.435 & -7.576\end{array}$

$-9.304$

$-2.153$

$-9.235$

$-9.244$

$-9.265$

$-9.253$

$-9.275$
$-2.068 \quad-7.167$

$\begin{array}{ll}-2.07 & -7.174\end{array}$

$-2.092 \quad-7.173$

$\begin{array}{ll}-2.092 & -7.161\end{array}$

$\begin{array}{ll}-2.156 & -7.119\end{array}$

$$
\begin{array}{rrr}
-9.004 & -1.403 & -7.601 \\
-8.935 & -1.201 & -7.734 \\
-8.944 & -1.202 & -7.742 \\
-8.969 & -1.358 & -7.611 \\
-8.960 & -1.363 & -7.597 \\
-8.985 & -1.412 & -7.573
\end{array}
$$




$\begin{array}{lrrrrrrrrr}\mathbf{1 c b m} & -9.015 & -1.481 & -7.534 & -9.262 & -2.111 & -7.151 & -8.980 & -1.423 & -7.557 \\ \mathbf{2 c a m}(\mathbf{s}) & -9.047 & -1.127 & -7.920 & -9.029 & -2.060 & -6.969 & -8.713 & -1.186 & -7.527 \\ \mathbf{2 c a m}(\mathbf{a}) & -9.082 & -1.138 & -7.944 & -9.048 & -2.066 & -6.982 & -8.733 & -1.198 & -7.535 \\ \mathbf{2 c b m}(\mathbf{s}) & -9.201 & -1.153 & -8.048 & -9.252 & -2.074 & -7.178 & -8.969 & -1.216 & -7.753 \\ \mathbf{2 c b m ( a )} & -9.222 & -1.149 & -8.073 & -9.259 & -2.075 & -7.184 & -8.965 & -1.220 & -7.745 \\ \mathbf{3 c a m}(\mathbf{s}) & -9.132 & -1.254 & -7.878 & -9.068 & -2.088 & -6.980 & -8.757 & -1.357 & -7.400 \\ \mathbf{3 c a m}(\mathbf{a}) & -9.082 & -1.224 & -7.858 & -9.042 & -2.083 & -6.959 & -8.734 & -1.338 & -7.396 \\ \mathbf{3 c b m}(\mathbf{s}) & -9.284 & -1.316 & -7.968 & -9.562 & -2.080 & -7.482 & -8.990 & -1.399 & -7.591 \\ \mathbf{3 c b m ( a )} & -9.255 & -1.292 & -7.963 & -9.268 & -2.101 & -7.167 & -8.993 & -1.387 & -7.606\end{array}$

\section{5(6)-Cl}

1c

2c(a)

Table 7

continued

$2 \mathrm{c}(\mathrm{s})$

$3 \mathbf{c}(\mathbf{a})$

$3 \mathbf{c}(\mathbf{s})$

1 cam

$1 \mathrm{cbm}$

2cam(s)

2cam(a)

$2 \operatorname{cbm}(s)$

$2 \operatorname{cbm}(a)$

$3 \mathrm{cam}(\mathrm{s})$

$3 \operatorname{cam}(a)$

$3 \operatorname{cbm}(s)$

$3 \operatorname{cbm}(a)$

$$
\begin{array}{lll}
-8.849 & -0.967 & -7.882
\end{array}
$$

$\begin{array}{lll}-8.974 & -0.600 \quad-8.374\end{array}$
$-9.063$

$-9.030$
$-0.839 \quad-8.224$

$-0.903 \quad-8.127$

$$
\begin{array}{lll}
-8.802 & -0.717 & -8.085 \\
-8.758 & -0.501 & -8.257
\end{array}
$$

${ }^{\mathrm{a}} \mathrm{n}=\mathrm{E}_{\mathrm{HOMO}}-\mathrm{E}_{\mathrm{LUMO}}$

\begin{tabular}{rrrrrrrrr}
-8.967 & -0.598 & -8.369 & -8.960 & -0.926 & -8.034 & -8.759 & -0.495 & -8.264 \\
-8.902 & -0.623 & -8.279 & -8.927 & -0.941 & -7.986 & -8.718 & -0.554 & -8.164 \\
-8.895 & -0.627 & -8.268 & -8.918 & -0.935 & -7.983 & -8.714 & -0.561 & -8.153 \\
-8.869 & -1.005 & -7.864 & -9.031 & -0.890 & -8.141 & -8.784 & -0.734 & -8.050 \\
-8.868 & -1.013 & -7.855 & -9.041 & -0.898 & -8.143 & -8.787 & -0.740 & -8.047 \\
-8.885 & -0.568 & -8.317 & -8.80 & -0.806 & -7.994 & -8.564 & -0.456 & -8.108 \\
-8.937 & -0.606 & -8.331 & -8.83 & -0.833 & -7.997 & -8.588 & -0.487 & -8.101 \\
-8.998 & -0.646 & -8.352 & -8.953 & -0.955 & -7.998 & -8.782 & -0.526 & -8.256 \\
-9.022 & -0.663 & -8.359 & -9.142 & -0.907 & -8.235 & -8.838 & -0.570 & -8.268 \\
-8.861 & -0.635 & -8.226 & -8.795 & -0.840 & -7.955 & -8.553 & -0.548 & -8.005 \\
-8.834 & -0.595 & -8.239 & -8.777 & -0.825 & -7.952 & -8.534 & -0.515 & -8.019 \\
-8.958 & -0.701 & -8.257 & -9.191 & -0.908 & -8.283 & -8.763 & -0.619 & -8.144 \\
-8.938 & -0.678 & -8.260 & -8.935 & -0.968 & -7.967 & -8.742 & -0.590 & -8.152 \\
\hline
\end{tabular}




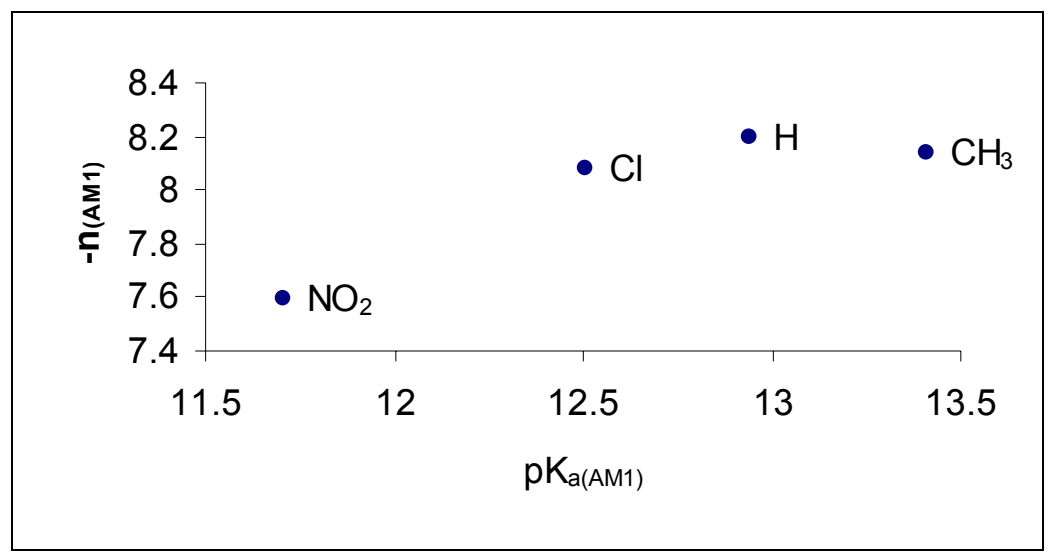

Figure 2. Plot of the aqueous phase AM1 calculated acidity constants, $\mathrm{pK}_{\mathrm{a}}$ (calc.), and nucleophilicity values $\mathrm{n}_{(\mathrm{AM} 1)}$, for studied molecules.

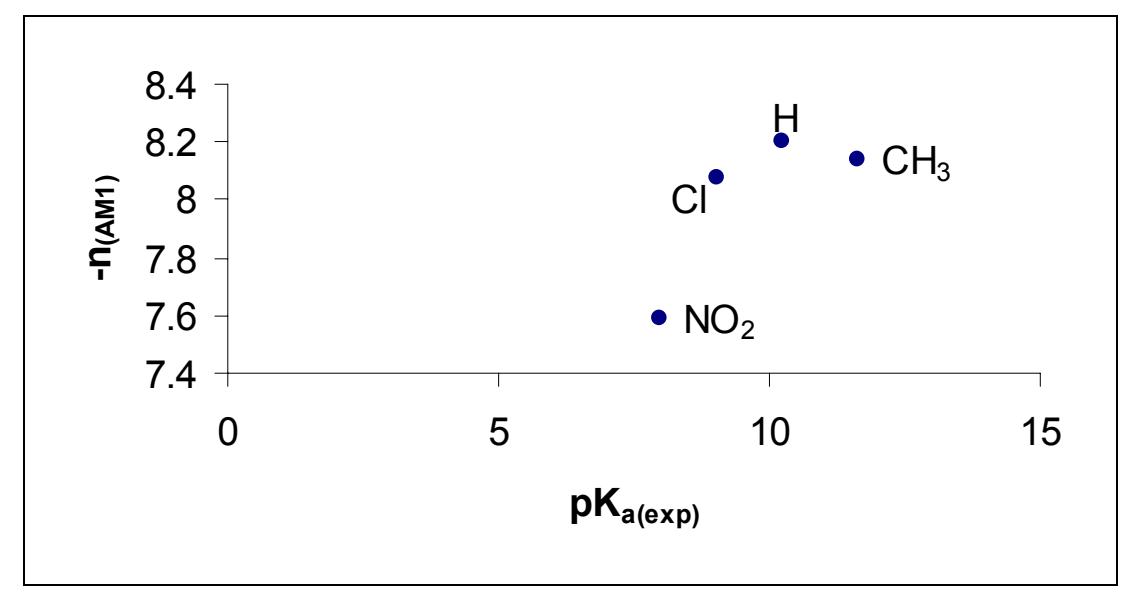

Figure 3. The plot of the aqueous phase experimental acidity constants, $\mathrm{pK}_{\mathrm{a}}(\mathrm{calc}$.), and nucleophilicity values $\mathrm{n}_{(\mathrm{AM} 1)}$, for studied molecules.

\section{Acknowledgement}

Our research group is greatly in debt to Eskişehir Osmangazi University for providing the MOPAC 2002 Cache program through Research Project number 200519010.

\section{References}

1. Acheson, R. M, Introduction to the Chemistry of Heterocyclic Compounds; Wiley, New York, (1976). 
2. Pelletier, S. V., Chemistry of the Alkaloids, Van Nostrand Reinhold Company; New York, (1970).

3. Woolley, D. W. J. Biol. Chem. 1944, 152, 225.

4. Klotz, I. M.; Mellody, M. J. Bact. 1948, 56, 253.

5. Öğretir, C.; Demirayak Ş. Chim. Acta Turc. 1990, 18, 120.

6. Öğretir, C.; Demirayak, Ş Chim. Acta Turc. 1990, 18, 285.

7. Öğretir, C.; Kanışkan, N. Turkish J. Chem. (Doğa), 1995, 19, 6

8. E Açıkkalp.; Yıldız, K.; Yarlıgan, S.; Öğretir C. J. Mol. Struct. (Theochem), 2001, 536, 155.

9. Öğretir, C ., Yarlıgan, S. J. Mol. Struct. (Theochem), 1996, 366, 227.

10. Öğretir C., Yarlıgan, S. J. Mol. Struct. (Theochem) , 1998, 425, 249.

11. Öğretir, C., Berber, H. J. Mol. Struct. (Theochem) , 2002, 577, 197.

12. Kanışkan. N.; Öğretir. C. J. Mol. Struct. (Theochem) , 2002, $584,45$.

13. Yarlıan, S.; Öğretir, C.; Kaynak, B.; Eseno,ğlu. E. J. Mol. Struct. (Theochem), 2002, 586, 9.

14. Katritzky, A. R.; Lagowsky, J. M. Adv. Heterocycl. Chem. 1963, 1, 339.

15. Katritzky, A. R.; Lagowsky, J. M. Adv. Heterocycl. Chem. 1964, 2, 1.

16. Elguero, I.; Marzin, C.; Katritzky, A. R.; Linda, P. Adv. Heterocyl. Chem. Suppl. 1976, 1, 1.

17. Kwiatkowski, J. S.; Pullman, B. Adv. Heterocyl. Chem., 1975, 18, 199.

18. Jones, R.A.; Whitmore, A. ARKIVOC, 2007, (xi), 114.

19. Martins, M. A. P.; Zanatta, N.; Bonacorso, H. G.; Rosa, F. A.; Claramunt, R. M.; Garcia, M. A.; Dolores, M.; Elguero, J. ARKIVOC, 2006, (iv), 29.

20. Poyraz, M.; Berber, H.; Ogretir, C. Submitted to ARKIVOC

21. Beak P. Acc. Chem. Res. 1977, 10, 186.

22. Beak, P.; Covington, J. B.; White, J. W. J. Org. Chem. 1980, 45, 1347.

23. Frank, J.; Katritzky, A. R. J. Chem. Soc., Perkin Trans. 2, 1976, 1428.

24. Cook, M. J.; Katritzky, A. R.; Linda, P.; Tack, R. D. J. Chem. Soc., Chem Commun., 1971, 510 .

25. Cook, M. J.; Katritzky, A. R.; Linda, P.; Tack, R. D. J. Chem. Soc., Perkin Trans. 2 1972, 1295; 1973, 1080.

26. Burnham. A. K.; Lee, J.; Schmalz, T. G.; Beak, P.; Flygare, W. H. J. Am. Chem. Soc. 1977, 99, 1836.

27. Ganellin C. R. in G. C. K. Roberts (ed.), Drug Action at Molecular Level University of Park Press, Baltimore, MD, 1977, Chapter 1.

28. J.J.P. Stewart MOPAC 2002 implemented in Cache Work System Pro., Version 6.1, Fujitsu Ltd.

29. COSMO, Klamt, A.; Schüümann, G. J. Chem. Soc., Perkin Trans. 1993, 2 , 799-805.

30. CS ChemOffice Pro for Microsoft Windows, Cambridge Scientific Computing Inc., 875 Massachusetts Avenue, Suite \&1, Cambridge MA, 02139, USA.

31. Cumper, C.W. N. G.; Pickering. D. J. Chem. Soc., Perkin Trans. 2 1972, 2045.

32. Prusner, P.; Sundaralingam M. Acta Crystallogr. Sect. B 1973,29, 2328. 
33. Speranza M. Adv. Heterocycl. Chem. 1985, 40, 25.

34. Brown, D. J. J. Chem. Soc. 1974, 1958.

35. Coarer J. Le; Wone M.; Broche A. , Ann. Sci. Univ. Dakar, 1961 ,6; 25.

36. Fleming I., Frontier Orbitals and Organic Reactions, pp 35-40, Wiley, Londo, 1976, 183190.

37. Hemmateenejad B.; Safarpour M. A.; Taghavi F. J. Mol. Struct. (Theochem), 2003, 635. 